\title{
Stationary field-aligned MHD flows at astropauses and in astrotails
}

\section{Principles of a counterflow configuration between a stellar wind and its interstellar medium wind ${ }^{\star}$}

\author{
D. H. Nickeler ${ }^{1,2,3}$, J. P. Goedbloed ${ }^{2,4}$, and H.-J. Fahr ${ }^{3}$ \\ 1 Astronomical Institute, AV ČR, Fričova 298, 25165 Ondřejov, Czech Republic \\ e-mail: nickeler@asu.cas.cz \\ 2 Astronomical Institute, University of Utrecht, Princetonplein 5, 3584 CC Utrecht, The Netherlands \\ Institute for Astrophysics and Extraterrestrial Research, Auf dem Hügel 71, 53121 Bonn, Germany \\ ${ }^{4}$ FOM-Institute for Plasma Physics, Edisonbaan 14, 3439 MN Nieuwegein, The Netherlands
}

Received 14 March 2005 / Accepted 14 March 2006

ABSTRACT

\begin{abstract}
Context. A stellar wind passing through the reverse shock is deflected into the astrospheric tail and leaves the stellar system either as a sub-Alfvénic or as a super-Alfvénic tail flow. An example is our own heliosphere and its heliotail.

Aims. We present an analytical method of calculating stationary, incompressible, and field-aligned plasma flows in the astrotail of a star. We present a recipe for constructing an astrosphere with the help of only a few governing parameters, like the inner Alfvén Mach number and the outer Alfvén Mach number, the magnetic field strength within and outside the stellar wind cavity, and the distribution of singular points (neutral points) of the magnetic field within these flows.

Methods. Within the framework of a one-fluid approximation, it is possible to obtain solutions of the governing MHD equations for stationary flows from corresponding static MHD equilibria, by using noncanonical mappings of the canonical variables. The canonical variables are the Euler potentials of the magnetic field of magnetohydrostatic equilibria. Thus we start from static equilibria determined by the distribution of magnetic neutral points, and assume that the Alfvén Mach number for the corresponding stationary equilibria is finite.

Results. The topological structure, i.e. the distribution of magnetic neutral points, determines the geometrical structure of the interstellar gas - stellar wind interface. Additional boundary conditions like the outer magnetic field and the jump of the magnetic field across the astropause allow determination of the noncanonical transformations. This delivers the strength of the magnetic field at every point in the astrotail/astrosheath region beyond the reverse shock.

Conclusions. The mathematical technique for describing such a scenario is applied to astrospheres in general, but is also relevant for the heliosphere. It shows the restrictions of the outer and the inner magnetic field strength in comparison with the corresponding Alfvén Mach numbers in the case of subalfvénic flows.
\end{abstract}

Key words. magnetohydrodynamics (MHD) - plasmas - methods: analytical - stars: winds, outflows - stars: magnetic fields

\section{Introduction}

\subsection{The scenario}

A wide range of literature is concerned with calculating stationary MHD flows for stellar magnetospheres, jets, stellar winds, and laboratory or general plasma configurations, see e.g. Chandrasekhar (1956), Tsinganos (1981), Lovelace et al. (1986), and Goedbloed \& Lifschitz (1997). These authors use one flux function to represent two components of the magnetic field and get a Grad-Shafranov type equation. This is a single nonlinear, partial differential equation for this magnetic flux function, and the method is restricted to 2D fields and flows. We apply a method that is not restricted to one flux function, but works with two flux functions of the magnetic fields.

Here we show that, under certain reasonable assumptions, it is possible to use a powerful transformation method for

* Appendices are only available in electronic form at http://www.edpsciences.org systematic modelling of the stellar wind region far away from the star itself. We apply this method to the special scenario of a stellar wind-interstellar medium (ISM) counterflow configuration (see Fig. 1 for the special case of the heliosphere). From this scenario, it is possible to estimate the pressure of the magnetic field, plasma pressure, and ram pressure, which are dynamically important for the ISM and therefore of high interest to astronomy (see e.g. Frisch 1993).

Beyond the region of the reverse shock ("Heliospheric Shock" in Fig. 1), the plasma of the stellar wind is decelerated. The magnetic field increases at the shock, so that a sub- or a super-Alfvénic plasma flow exists in the downstream direction. A contact surface forms between the two different flows. This separatrix is called the astropause. Scherer et al. (1994) and Fahr et al. (1993) showed that the bulk flow in the downwind direction can be assumed to be incompressible for small Mach numbers. This holds even more for field aligned flows, since the field lines act as quasi-isothermals. As the decelerated stellar wind has to adapt to the conditions of an outer magnetized Very Local 


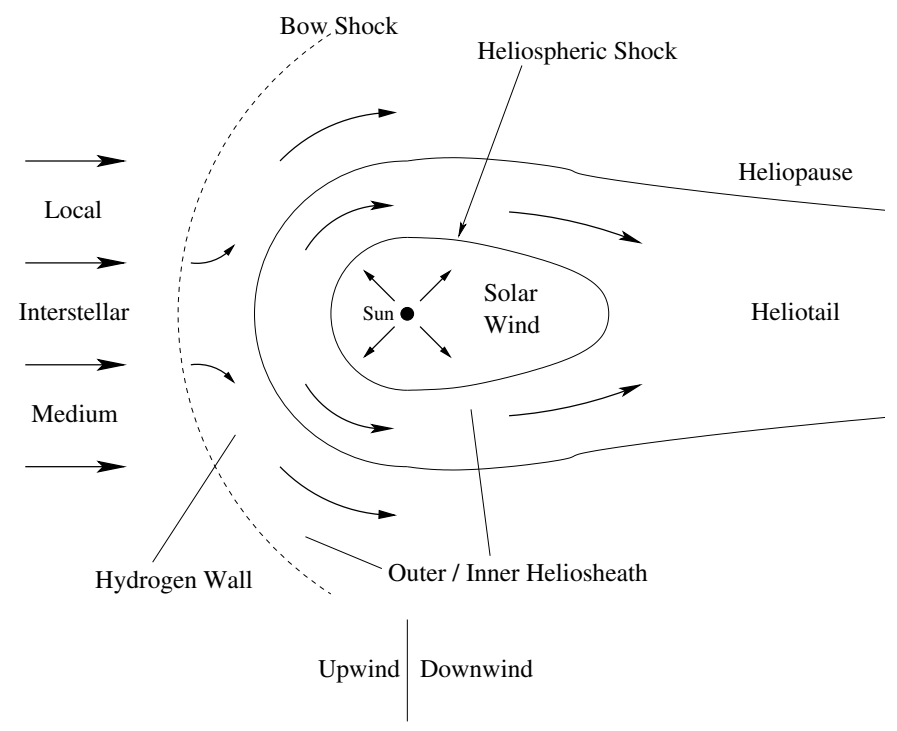

Fig. 1. Sketch of the heliosphere shown as a special example of an astrosphere.

InterStellar Medium (VLISM), a tangential discontinuity forms. This is called the astropause (AP, indicated by "Heliopause" in Fig. 1), which stretches out in the downwind direction, so that the whole structure has a tail-like shape (see Fig. 1). Between the termination shock (TS, the heliospheric shock in Fig. 1) and the $\mathrm{AP}$ an inner astrosheath region extends into the astrotail, similar to the Earth's magnetotail. In the magnetotail, the MHD quantities depend mainly on the direction perpendicular to the tail axis (e.g. Schindler 1972). In view of the evident similarities, we here apply a similar description to astrotails. We show that there is a strong correlation between the flow, especially its Alfvén Mach number inside and outside the astropause region, and the current density in the vicinity of the astropause. This correlation also sets restrictions on the relation between outer and inner values of the magnetic field and the Alfvén Mach numbers.

\subsection{Model considerations and existing models}

Astrospheric models can be divided into two kinds, analytical and numerical, but there is a gap between these two. In most cases, the analytical models are only hydrodynamical or purely magnetic models (e.g. Parker 1961). Parker's article only treats purely hydrodynamic models of the subsonic counterflow or of an unmagnetized stellar wind that blows into a magnetohydrostatic interstellar environment (Parker 1961). His models connect parameters, like the pressure of the magnetic field and the thermal pressure, with the shape of the modelastrospheres. Another analytical model was the first superAlfvénic MHD model suggested for the SW-ISM interaction, which is based on the thin-layer (hypersonic) approximation (Baranov \& Krasnobaev 1971). In contrast to the model of Parker or Baranov and Krasnobaev the geometry of our model, presented here in this article, does not explicitly depend on the Alfvén Mach number or the usual Mach number of the flow. We propose a different point of view in this article by emphasizing the connection between topological aspects of the magnetic field structure and the geometrical shape of the astropause.

Other analytical models have been calculated for pressure equilibrium (Newtonian approximation by Fahr et al. 1988) or for those configurations where the plasma cavity is a finite ellipsoid, and the plasma has to leave the astrosphere by diffusion (Neutsch \& Fahr 1983). Other authors use the set of MHD equations, but prefer solving the ideal Ohm's law and neglecting the Lorentz force in the Euler equation (see the kinematical approach by Suess \& Nerney 1992, 1990; Nerney et al. 1991). Up to now no analytical and exact solutions of the MHD equations exist for this scenario. This motivated us to consider models that do not depend on mathematical approximations, but where (additional) physical approximations are taken into account. For example, Imai (1960) analysed field-aligned flows and calculated approximative solutions. However, we take into account that a flow that has passed a shock is likely to develop a sub-sonic/sub-Alfvénic flow with a negligible compressibility along the field- and streamlines. The argument in Scherer et al. (1994) and Fahr et al. (1993) is that the low Mach number does not provide high compressibility rates. Another argument for "incompressibility" is that in a tail that is symmetric with respect to the tail axis, the far-away field- and streamlines are nearly one-dimensional. This implies a one-dimensional dependence of the physical values perpendicular to this axis, see Schindler (1972), so that the density is approximately constant on field lines.

Webb et al. (1994) analysed two-dimensional MHD flows and discussed the properties of transsonic flows. We focus on the relation between the Alfvén Mach number $M_{\mathrm{A}}$ and the electric current density, which can be given by the jump of the two magnetic fields across the boundary of the astropause of, in principle, $3 \mathrm{D}$ configurations. We also focus on topological and geometrical questions with respect to the boundary between two magnetized flows.

Simulation results may also be reliable, although they can deliver unphysical results, even when stable algorithms are used. For example, Linde et al.(1998) discovered magnetic diffusion in their simulation domain, although they used an ideal MHD code. Evidently, numerical magnetic reconnection is taking place in some region of their domain of calculation.

Numerical reconnection cannot take place in our treatment since we calculate exact and analytical solutions of the ideal MHD equations with finite width of the astropause current sheet. We describe smooth flows without shocks, i.e. without nontangential discontinuities, only. In our approach, we have restricted the analysis to tangential discontinuities. The possibility of Alfvénic discontinuities is discussed by Smith (2001) for the heliospheric current sheet. Thus, we close the gap between the older analytical, but simplified, treatments of the astrophysical counterflow problem and the sophisticated numerical models. We find principles for these counterflow scenarios that should also hold for simulations having fewer physical simplifications. Our aim is to calculate analytical and exact solutions of the stationary nonlinear MHD equations for stellar wind tails, extending the approximative analytical models discussed above.

The paper is structured as follows. In Sect. 2, we present the equations to be solved for our scenario and the special method used to solve them. In Sect. 3, we show how this method works in the two-dimensional case, which is a simplification of our model, focussing on the method and the basic principles of MHD counterflows. In Sect. 4, we show how to construct the flow and its stream lines, i.e. the magnetic field structure. This gives us the pattern of such MHD flows. We use the simplest magnetohydrostatic equilibria, viz. potential fields. The reason for it will be given in Sect. 4. Section 5 discusses the dependence on the symmetry and boundary conditions for the asymptotical one-dimensional case, which is interesting for tail-like structures of the magnetic field. In addition, we present some 
two-dimensional tail models. Discussion and conclusions are given in Sect. 6.

\section{Stationary states in incompressible and ideal MHD}

The set of equations that must be solved to get incompressible ideal MHD flows consists of the mass continuity Eq. (1), the Euler or momentum equation with isotropic pressure $P(2)$, the induction equation including the ideal Ohm's law (3), Ampère's law (4), the initial condition for the magnetic field (5), and the condition for incompressibility (6):

$$
\begin{aligned}
\boldsymbol{\nabla} \cdot(\rho \boldsymbol{v}) & =0, \\
\rho(\boldsymbol{v} \cdot \boldsymbol{\nabla}) \boldsymbol{v} & =\boldsymbol{j} \times \boldsymbol{B}-\boldsymbol{\nabla} P, \\
\boldsymbol{\nabla} \times(\boldsymbol{v} \times \boldsymbol{B}) & =\mathbf{0}, \\
\boldsymbol{\nabla} \times \boldsymbol{B} & =\mu_{0} \boldsymbol{j}, \\
\boldsymbol{\nabla} \cdot \boldsymbol{B} & =0, \\
\boldsymbol{\nabla} \cdot \boldsymbol{v} & =0 .
\end{aligned}
$$

Due to the incompressibility, the mass continuity equation can be written as $\boldsymbol{v} \cdot \boldsymbol{\nabla} \rho=0$, so that the density is constant on streamlines. If we now introduce the auxilliary flow vector $w:=\sqrt{\rho} v$ and the Bernoulli pressure $\Pi:=P+\frac{1}{2} w^{2}$, we can write the above equations as

$$
\begin{aligned}
\boldsymbol{\nabla} \cdot \boldsymbol{w} & =0, \\
\boldsymbol{\nabla} \Pi & =\frac{1}{\mu_{0}}(\boldsymbol{\nabla} \times \boldsymbol{B}) \times \boldsymbol{B}-(\boldsymbol{\nabla} \times \boldsymbol{w}) \times \boldsymbol{w}, \\
\boldsymbol{\nabla} \times\left(\frac{1}{\sqrt{\rho}} \boldsymbol{w} \times \boldsymbol{B}\right) & =\mathbf{0}, \\
\boldsymbol{\nabla} \cdot \boldsymbol{B} & =0 .
\end{aligned}
$$

Hence, the momentum equation Eq. (8) is written such that the analogy with magnetohydrostatic equilibria, $\nabla P=$ $\mu_{0}^{-1}(\nabla \times \boldsymbol{B}) \times \boldsymbol{B}$, is evident.

\subsection{Field-aligned flows}

The stationary equilibria should be constructed such that they tend to be stable in order to use them as stationary background fields in very turbulent and time-dependent stellar winds. In analytical works (e.g. Suess \& Nerney 1992, and references therein), this problem is often treated kinematically, which means that the Lorentz force is ignored. These authors find strong amplification of convected magnetic fields in the so-called upwind direction, which is the direction from which the interstellar medium is flowing towards the star. In this direction they identified a cone of 30 degrees (where the star is sitting at the top of the cone), in which their kinematical approach is invalid. Such velocity fields with a strong perpendicular component to the magnetic field have a saddle-point structure in linear stability analysis (Hameiri 1998) and are, therefore, likely to develop ideal MHD instabilities.

Hameiri found that a variational principle does not lead to a stability criterion if velocity and magnetic field are not aligned, because the used functional has only stationary points, but has no minimum. Hameiri (1998) suggests that the lack of a minimum is due to the presence of ballooning modes. In fact assuming the incompressible limit, the equilibrium velocity field has to be subAlfvénic to ensure the existence of a minimum. Thus, to calculate magnetohydrodynamic configurations that should "survive" long enough to represent a quasi-stationary state of the stellar wind flow, it is necessary to assume field aligned flows. This ensures that these configurations can really exist in nature, i.e. that they are sufficiently long-lived to be represented as stationary MHD flows. This would not be the case with strong perpendicular components of the flow with respect to the magnetic field, since those would lead to quick ideal MHD instabilities. Thus, models where magnetic and flow field are not (approximately) aligned cannot exist in nature without showing strong time dependency. Since strong time-dependent MHD flows, instabilities, and shocks do occur in the corotating interaction regions of the solar system, the validity of our models is restricted to those regions far outside the termination shock. There, incompressible and field-aligned flows are good approximations of the real outflows of stars, at least when they are sufficiently far away from the stellar surface ${ }^{1}$.

From this point of view, to lower the risk of instability, it is expedient to make the simplifying assumption of field aligned flow, i.e.

$\boldsymbol{w}= \pm \frac{M_{\mathrm{A}}}{\sqrt{\mu_{0}}} \boldsymbol{B}$

where $M_{\mathrm{A}}$ is the Alfvén Mach number. This equation fulfills the induction Eq. (9) automatically. The sign on the righthand side of Eq. (11) is to be understood in the framework of the transformation method introduced in the next subsection. With this assumption, we can skip Eq. (9), and from Eqs. (11), (7), and (10) it follows directly that

$$
\boldsymbol{w} \cdot \boldsymbol{\nabla} M_{\mathrm{A}}=0, \quad \text { and } \quad \boldsymbol{B} \cdot \boldsymbol{\nabla} M_{\mathrm{A}}=0 .
$$

Therefore, the mass density $\rho$ and the Alfvén Mach number are constant on field lines, but they can vary perpendicular to them. In conclusion, we have to solve the following system of equations:

$$
\begin{aligned}
\boldsymbol{B} \cdot \boldsymbol{\nabla} M_{\mathrm{A}} & =0 \\
\nabla \Pi & =\frac{1}{\mu_{0}}\left(1-M_{\mathrm{A}}^{2}\right)(\boldsymbol{\nabla} \times \boldsymbol{B}) \times \boldsymbol{B}-\frac{|\boldsymbol{B}|^{2}}{2 \mu_{0}} \nabla\left(1-M_{\mathrm{A}}^{2}\right), \\
\boldsymbol{\nabla} \cdot \boldsymbol{B} & =0 .
\end{aligned}
$$

This system determines the unknowns $\boldsymbol{B}, M_{\mathrm{A}}$ and $\Pi$. In the next subsection we present a method of solving the general threedimensional problem given by the system of Eqs. (13)-(15) by means of a noncanonical transformation. Later on, we explicitly calculate two-dimensional equilibria in order to highlight the main properties that are important for understanding relaxed, magnetized, stellar tail flows. The reduction of this system to one magnetic flux function (e.g. Tsinganos 1981; Goedbloed \& Lifschitz 1997) will be done in Sect. 3.

\subsection{Euler potential representation and noncanonical transformations}

In most cases in the literature (e.g. Chandrasekhar 1956; Tsinganos 1981; Lovelace et al. 1986; Goedbloed \& Lifshitz 1997), the problem of solving the stationary MHD equations is reduced to equations similar to the Grad-Shafranov equation (GSE, see e.g. Grad \& Rubin 1958) by introducing twodimensional flux functions for the magnetic field. Here, we give

\footnotetext{
1 This is also valid, if we focus our view either on the classical (subsonic unmagnetized flow, radially extrapolated to the origin) Parker flow or on the Parker spiral magnetic field, which we use as a geometrical pattern for calculating flows and magnetic fields in the next sections.
} 
a short introduction to a different method that allows us to calculate also fields that could be three dimensional.

In 1984, Zwingmann showed the similarity between magnetohydrostatic (MHS) equilibria and stationary MHD equilibria with incompressible, field-aligned flows. Later, this theory was improved by Gebhardt \& Kiessling (1992), and subsequently used by Petrie \& Neukirch (1999) for modelling sunspot magnetic fields with plasma flow. We briefly recapitulate the transformation method used in the cited papers in order to facilitate the analysis of our astrospheric model.

In general, non-ergodic magnetic fields can be represented by using Euler potentials (see e.g. Kruskal \& Kulsrud 1958 or D'haeseleer 1990, and references therein). The magnetic fields of MHS equilibria can also be represented by using Euler potentials, writing

$\boldsymbol{B}_{S}=\boldsymbol{\nabla} f \times \nabla g$,

where the Euler or Clebsch potentials $f$ and $g$ are scalar functions of $x, y, z$ in general. Here, and in the following, the subscript $S$ will be used to indicate magnetohydrostatic equilibrium quantities. The MHS equations can now be written as canonical Hamiltonian equations:

$$
\begin{array}{r}
\frac{\partial P_{S}}{\partial f}=\boldsymbol{j}_{S} \cdot \nabla g, \\
-\frac{\partial P_{S}}{\partial g}=\boldsymbol{j}_{S} \cdot \boldsymbol{\nabla} f,
\end{array}
$$

with the canonical variables $f$ and $g$ and the arc length $s$ along the current $j_{S}$ (see Schindler 1979). The MHS field $\boldsymbol{B}_{S}$ can now be mapped to a new field $\boldsymbol{B}$ by performing the transformation,

$$
\begin{aligned}
& f=f(\alpha, \beta) \\
& g=g(\alpha, \beta)
\end{aligned} \quad \begin{aligned}
& \alpha=\alpha(f, g) \\
& \beta=\beta(f, g)
\end{aligned}
$$

where the derivatives as well as the inverse mappings and its derivatives, are assumed to exist. Then, there is a relationship between the old (static) field $\boldsymbol{B}_{S}$ and the new field $\boldsymbol{B}$, which can be interpreted as a stationary field:

$$
\boldsymbol{B}_{S}=\boldsymbol{\nabla} f \times \boldsymbol{\nabla} g=[f, g]_{\alpha, \beta} \boldsymbol{\nabla} \alpha \times \boldsymbol{\nabla} \beta \equiv[f, g]_{\alpha, \beta} \boldsymbol{B},
$$

where the Poisson bracket $[f, g]_{\alpha, \beta}$ is the Jacobian of the transformation Eq. (19). If the Poisson bracket $[f, g]_{\alpha, \beta}=1$, it can be seen from Eq. (20) that only a change of the potentials but no real active transformation has taken place, so that the magnetic field has not been changed. Therefore, $\alpha$ and $\beta$ would also be canonical variables for the field $\boldsymbol{B}_{S}$, and the mapping from $\boldsymbol{B}_{S}$ to $\boldsymbol{B}$ would be a canonical transformation, which does not produce new physics. However, if the Poisson bracket $[f, g]_{\alpha, \beta} \neq 1$, then the magnetic field $\boldsymbol{B}$ has to be interpreted in a different way, as it is not possible to identify it as a magnetic field of a static equilibrium.

The similarity between MHS, Eq. (21) below, and MHD, Eq. (14), can be seen by inspecting the original momentum balance equation of the MHS field, when we insert Eq. (19) into $\boldsymbol{\nabla} P_{S}=j_{S} \times \boldsymbol{B}_{S}$, which leads to

$$
\begin{aligned}
\boldsymbol{\nabla} P_{S}= & \frac{1}{\mu_{0}}(\boldsymbol{\nabla} \times(\boldsymbol{\nabla} f \times \boldsymbol{\nabla} g)) \times(\boldsymbol{\nabla} f \times \boldsymbol{\nabla} g) \\
= & \frac{[f, g]_{\alpha, \beta}^{2}}{\mu_{0}} \boldsymbol{\nabla} \times(\boldsymbol{\nabla} \alpha \times \boldsymbol{\nabla} \beta) \times(\boldsymbol{\nabla} \alpha \times \boldsymbol{\nabla} \beta) \\
& -\frac{|\boldsymbol{\nabla} \alpha \times \boldsymbol{\nabla} \beta|^{2}}{2 \mu_{0}} \nabla[f, g]_{\alpha, \beta}^{2} .
\end{aligned}
$$

Consequently, the relation between the Poisson bracket and the Alfvén Mach number is given by

$0<\left([f, g]_{\alpha, \beta}\right)^{2}:=1-M_{\mathrm{A}}^{2}$

for purely sub-Alfvénic flows, and by

$0<\left([f, g]_{\alpha, \beta}\right)^{2}:=M_{\mathrm{A}}^{2}-1$

for purely super-Alfvénic flows.

Setting $\boldsymbol{B}=\mathbf{0}$ in Eq. (8), which describes a purely incompressible stationary hydrodynamical flow, Gebhardt \& Kiessling (1992) noted the similarity between Eqs. (8) and (21). This can be seen if the auxiliary flow field $w$ is also represented by Euler potentials. Then a mapping from a known solution of stationary incompressible hydrodynamics to a stationary incompressible, super-Alfvénic field-aligned flow is possible, if $\left([f, g]_{\alpha, \beta}\right)^{2}>1$. It is possible to map a known solution of the MHS equations by means of a transformation with $\left([f, g]_{\alpha, \beta}\right)^{2}<1$ to an incompressible MHD equilibrium with a field-aligned sub-Alfvénic flow. Thus for every incompressible field-aligned sub-Alfvénic flow, it is possible to find a mapping onto a MHS equilibrium. Alternatively, it is also possible to take a different look at the problem by focussing on the "current-generating" transformation of a given MHS equilibrium, as we are interested in the astropause current sheet. This works as follows: from Eqs. (21) and (23) it is obvious that for a certain transformation, e.g. given by a sub-Alfvénic flow with the Jacobian squared $\left([f, g]_{\alpha, \beta}\right)^{2}<1$

$\boldsymbol{w}_{\text {sub }}=\operatorname{sign}\left[[f, g]_{\alpha, \beta}\right] \frac{\sqrt{1-\left([f, g]_{\alpha, \beta}\right)^{2}}}{\sqrt{\mu_{0}}} \boldsymbol{B}$,

there exists a corresponding super-Alfvénic solution for the flow fields:

$\boldsymbol{w}_{\text {super }}=\operatorname{sign}\left[[f, g]_{\alpha, \beta}\right] \frac{\sqrt{\left([f, g]_{\alpha, \beta}\right)^{2}+1}}{\sqrt{\mu_{0}}} \boldsymbol{B}$.

This has to obey the following restriction

$$
\begin{aligned}
0<[f, g]_{\alpha, \beta}^{2} & =M_{\mathrm{A}, \text { super }}^{2}-1 \equiv 1-M_{\mathrm{A}, \text { sub }}^{2}<1 \\
\Rightarrow \quad M_{\mathrm{A}, \text { super }}^{2} & \equiv 2-M_{\mathrm{A}, \text { sub }}^{2},
\end{aligned}
$$

to be satisfied at every point in space, while the magnetic field is the same as in the sub-Alfvénic case: $\boldsymbol{B}_{\text {super }} \equiv \boldsymbol{B}_{\text {sub }} \equiv \boldsymbol{B}$.

Therefore, it is not guaranteed for all Poisson brackets, i.e. transformations, that sub-Alfvénic solutions exist, but for a given sub-Alfvénic solution $\left([f, g]_{\alpha, \beta}\right)^{2}<1$ a corresponding superAlfvénic MHD flow exists with $1<M_{\mathrm{A} \text {,super }}^{2}<2$. For these flows, the magnetic field of the underlying MHS equilibrium magnetic field will be amplified:

$|\boldsymbol{B}| \equiv\left|\boldsymbol{B}_{\text {super }}\right|=\left|\frac{\boldsymbol{B}_{S}}{\sqrt{M_{\mathrm{A}, \text { super }}^{2}-1}}\right|>\left|\boldsymbol{B}_{S}\right|$.

It is also necessary for the super-Alfvénic case that the thermal or plasma pressure $\Pi_{\text {super }}$ is "inverted" to regain the similarity between Eqs. (14) and (21),

$\nabla P_{S} \equiv \nabla \Pi_{\text {sub }}$ 
changed to

$\boldsymbol{\nabla} P_{S} \equiv \boldsymbol{\nabla}\left(-\Pi_{\text {super }}\right)$,

where $\Pi_{\text {sub }}$ is the sub- and $\Pi_{\text {super }}$ is the corresponding superAlfvénic Bernoulli pressure. Integration of these equations leads to

$P_{S}=\Pi_{\text {sub }}+\Pi_{0}$,

$P_{S}=-\Pi_{\text {super }}+\Pi_{1}$,

where $\Pi_{0}$ and $\Pi_{1}$ are integration constants. It follows from Eqs. (31) and (32) that

$P_{\text {super }}=\Pi_{1}-\Pi_{0}-\left(\frac{|\boldsymbol{B}|^{2}}{2 \mu_{0}}+P_{\text {sub }}\right)$.

All the above-mentioned relations and considerations are also valid for the case $M_{\mathrm{A} \text {,super }}^{2}-1=\left([f, g]_{\alpha, \beta}\right)^{2}>1$. Only Eqs. (26), (27), (31), and (33) are not valid then, along with the inequality Eq. (28). However, what does remain valid is that the magnetic field of the underlying MHS equilibrium can be amplified or weakened:

$\boldsymbol{B} \equiv \boldsymbol{B}_{\text {super }}=\frac{\boldsymbol{B}_{S}}{\sqrt{M_{\mathrm{A}, \text { super }}^{2}-1}}$,

with domains where $|\boldsymbol{B}|$ can be larger, and domains where $|\boldsymbol{B}|$ can be smaller than $\boldsymbol{B}_{S}$.

We want to focus on purely sub-Alfvénic flows. In this case the transformation equations from the static to the stationary fields can be written as

$$
\begin{array}{rlrl}
\boldsymbol{B}_{S}=\boldsymbol{\nabla} f \times \boldsymbol{\nabla} g & \longmapsto & \boldsymbol{B}=\boldsymbol{\nabla} \alpha \times \boldsymbol{\nabla} \beta=\frac{\boldsymbol{B}_{S}}{\sqrt{1-M_{\mathrm{A}}^{2}}}, \\
P_{S}=P_{S}(f, g) & \longmapsto & P=P_{S}-\frac{1}{2} \rho|\boldsymbol{v}|^{2}, \\
\boldsymbol{v} & =0 & \longmapsto & \boldsymbol{v}=\frac{M_{\mathrm{A}} \boldsymbol{B}_{S}}{\sqrt{\mu_{0} \rho\left(1-M_{\mathrm{A}}^{2}\right)}} .
\end{array}
$$

We are interested in the fact that an astrosphere is terminated by a boundary between two different magnetic fields. For the magnetic field, this boundary is a tangential discontinuity or an encounter of two magnetic fields with a large gradient across that boundary. This boundary, the astropause, can therefore be regarded as a current layer, so we need additional information. We can deduce that the electric current density is also transformed by

$$
\begin{aligned}
& \mu_{0} \boldsymbol{j}= \boldsymbol{\nabla} \times(\boldsymbol{\nabla} \alpha \times \boldsymbol{\nabla} \beta) \\
&= \boldsymbol{\nabla}[\alpha, \beta]_{f, g} \times(\boldsymbol{\nabla} f \times \boldsymbol{\nabla} g)+[\alpha, \beta]_{f, g} \boldsymbol{\nabla} \times(\boldsymbol{\nabla} f \times \boldsymbol{\nabla} g) \\
&= \boldsymbol{\nabla}[\alpha, \beta]_{f, g} \times \boldsymbol{B}_{S}+\mu_{0}[\alpha, \beta]_{f, g} \boldsymbol{j}_{S} \\
& \stackrel{M_{\mathrm{A}}<1}{=} \frac{\operatorname{sign}\left[[\alpha, \beta]_{\mathrm{f}, \mathrm{g}}\right] M_{\mathrm{A}}}{\sqrt{\left(1-M_{\mathrm{A}}^{2}\right)^{3}}}\left[\nabla f\left(\nabla M_{\mathrm{A}} \cdot \boldsymbol{\nabla} g\right)-\boldsymbol{\nabla} g\left(\boldsymbol{\nabla} M_{\mathrm{A}} \cdot \boldsymbol{\nabla} f\right)\right] \\
&+\frac{\operatorname{sign}\left[[\alpha, \beta]_{\mathrm{f}, \mathrm{g}}\right]}{\sqrt{1-M_{\mathrm{A}}^{2}}} \nabla \times(\nabla f \times \nabla g)
\end{aligned}
$$

which implies that even in the case of a static equilibrium with vanishing current density, i.e. a potential field, one gets a stationary equilibrium with a non-vanishing current,

$\boldsymbol{j}=\frac{\operatorname{sign}\left[[\alpha, \beta]_{\mathrm{f}, \mathrm{g}}\right] M_{\mathrm{A}}}{\mu_{0} \sqrt{\left(1-M_{\mathrm{A}}^{2}\right)^{3}}}\left[\boldsymbol{\nabla} f\left(\boldsymbol{\nabla} M_{\mathrm{A}} \cdot \boldsymbol{\nabla} g\right)-\boldsymbol{\nabla} g\left(\boldsymbol{\nabla} M_{\mathrm{A}} \cdot \boldsymbol{\nabla} f\right)\right]$.
In addition to the previous works by the mentioned authors (Gebhardt \& Kiessling 1992; Petrie \& Neukirch 1999), we have found that the flow and the current are strongly correlated by means of the Alfvén Mach number.

\section{Two-dimensional equilibria}

To get exact and analytical equilibria, we restrict our view to two-dimensional equilibria since only symmetric equilibria are known in infinite domains, see e.g. Tsinganos (1982). There are no analytical and exact 3D MHD equilibria known that are bounded and that extend throughout the whole 3D space. Therefore, we assume from now on that $f=A$, where $A$ is a function of $x$ and $y$, and that $g=z$, so that we get the GSE (see e.g. Grad \& Rubin 1958):

$\Delta A=-\mu_{0} \frac{\mathrm{d} P_{S}}{\mathrm{~d} A}=-\mu_{0} j_{z S}$

and a relation between the Alfvén Mach number and the derivative of $\alpha$ :

$M_{\mathrm{A}}^{2}=1-\frac{1}{\alpha^{\prime}(A)^{2}} \quad \Leftrightarrow \quad\left(\alpha^{\prime}(A)\right)^{2}=\left(\frac{\mathrm{d} \alpha}{\mathrm{d} A}\right)^{2}=\frac{1}{1-M_{\mathrm{A}}^{2}}$.

The current density for the stationary equilibrium can then be expressed by

$\Delta \alpha=-\mu_{0} j_{z}=\boldsymbol{\nabla} \cdot(\boldsymbol{\nabla} \alpha)=\alpha^{\prime \prime}(A)|\nabla A|^{2}+\alpha^{\prime}(A) \Delta A$.

The calculation of the current can also be derived from Eqs. (38) and (39) where we have transformed the current density for general 3D equilibria following Ampère's law, setting $f=A$ and $g=z$. This is reasonable, if a domain in the vicinity of the equatorial plane of the star is to be represented by the calculated equilibria.

\section{The pattern of the flow field and the magnetic field: potential fields as magnetohydrostatic equilibra}

We show in this section that the distribution of (virtual or real) magnetic neutral points, which are also stagnation points if we assume a finite Alfvén Mach number, determines the global topological and geometrical structure of the astrosphere. In the linear case, there is a relation between the magnetic multipole moments and the neutral points that will be given later on. But also for a more complicated class of nonlinear solutions of the MHS equations, it is possible to find similar relations. Those will be discussed in a future paper.

There are several reasons for using potential fields as origins for our mappings. They are much simpler to handle than nonlinear MHS fields in the framework of our solution technique, where the nonlinearity of the MHD equations is handled by a nonlinear mapping technique. Hence, one only has to solve a linear partial differential equation, whereas the nonlinearity is hidden in a nonlinear algebraic equation. In addition, potential fields have a highly stable character as they have no free magnetic energy, although it is not known if stability is conserved after the mapping onto a stationary equilibrium (see Petrie \& Neukirch 1999). Also, the connection between the neutral point distribution of the magnetic field and the global structure of the magnetic and the velocity fields can clearly be seen.

Another reason is that there should be at least one saddle point (the so-called X-point) in this counterflow configuration. 
Amongst $(N+1)$ null points, at least one point must exist in the vicinity of the nose of the astropause (at the stagnation point) for which the eigenvalues of the Jacobian of the linearized magnetic field $^{2}$,

$\left(\begin{array}{ll}\frac{\partial B_{x}}{\partial x} & \frac{\partial B_{x}}{\partial y} \\ \frac{\partial B_{y}}{\partial x} & \frac{\partial B_{y}}{\partial y}\end{array}\right)\left(\begin{array}{l}x \\ y\end{array}\right)=\left(\begin{array}{l}B_{1 x} \\ B_{1 y}\end{array}\right)$

take the form

$\lambda= \pm \lambda_{1}, \lambda_{1} \in \mathbb{R}$.

With the Grad Shafranov equation

$\Delta A=-\mu_{0} \frac{\mathrm{d} P}{\mathrm{~d} A}=J(A) \Rightarrow \frac{\partial^{2} A}{\partial y^{2}}=J(A)-\frac{\partial^{2} A}{\partial x^{2}}$,

where $J(A)$ is the current function, we can write the linearized magnetic field as

$\left(\begin{array}{cc}\frac{\partial^{2} A}{\partial x \partial y} & J(A)-\frac{\partial^{2} A}{\partial x^{2}} \\ -\frac{\partial^{2} A}{\partial x^{2}} & -\frac{\partial^{2} A}{\partial x \partial y}\end{array}\right)\left(\begin{array}{l}x \\ y\end{array}\right)=\left(\begin{array}{l}B_{1 x} \\ B_{1 y}\end{array}\right)$

The properties of the eigenvalues of the Jacobian of the magnetic field determine whether the neutral (or stagnation) point is a point with which one can define a separatrix curve (or surface, see Arnold 1992; Reitmann 1996). The separatrix is a border surface between two different regions of a flow, or of a magnetic field that separates a vector field in areas of different topological connections. To get information about the topological structure of the magnetic field, we calculate

$\operatorname{Det}\left(\begin{array}{cc}\frac{\partial^{2} A}{\partial x \partial y}-\lambda J(A)-\frac{\partial^{2} A}{\partial x^{2}} \\ -\frac{\partial^{2} A}{\partial x^{2}} & -\frac{\partial^{2} A}{\partial x \partial y}-\lambda\end{array}\right)=0$

$\Rightarrow \quad \lambda^{2}-\left(\frac{\partial^{2} A}{\partial x \partial y}\right)^{2}-\left(\frac{\partial^{2} A}{\partial x^{2}}\right)^{2}+J(A) \frac{\partial^{2} A}{\partial x^{2}}=0$.

Therefore, we obtain the following eigenvalues:

$$
\begin{aligned}
\lambda & = \pm\left[\left(\frac{\partial^{2} A}{\partial x \partial y}\right)^{2}+\left(\frac{\partial^{2} A}{\partial x^{2}}\right)^{2}-J(A) \frac{\partial^{2} A}{\partial x^{2}}\right]^{\frac{1}{2}} \\
& = \pm\left[\left(\frac{\partial^{2} A}{\partial x \partial y}\right)^{2}+2\left(\frac{\partial^{2} A}{\partial x^{2}}\right)^{2}+\frac{\partial^{2} A}{\partial y^{2}} \frac{\partial^{2} A}{\partial x^{2}}\right]^{\frac{1}{2}} .
\end{aligned}
$$

For two conjugate complex solutions in $2 \mathrm{D}$, we obtain $\lambda=$ $\pm \lambda_{1} \in \mathbb{C}$, i.e. only two purely imaginary values exist. If only two real eigenvalues exist, there is a saddle point (also called $\mathrm{X}$-point), which is necessary for the existence of a separatrix and which guarantees that there is a boundary surface between two distinct areas of the flow; i.e. an astropause exists. If $A$ is a

${ }^{2}$ The global coordinates $(X, Y)$ are replaced here by $(x, y)=(X-$ $\left.X_{S}, Y-Y_{S}\right)$, with $\left(X_{S}, Y_{S}\right)$ being the coordinates of the null point. potential field, i.e. $\Delta A=J(A)=0$, then the null point is a saddle point, as can be seen from Eq. (49). If the eigenvalues at the null point are purely imaginary, then, depending on the absolute value, a centre (a so-called $O$-point) exists with topological circles as fieldlines, or a so-called focus with spiral fieldlines. The last case is not found for solenoidal vector fields, since the trace of the Jacobian matrix vanishes for them.

\subsection{Potential fields}

We now have to calculate the flow pattern, i.e. the magnetic field pattern. This can be done by calculating the most simple and stable magnetic fields, namely potential fields. We construct solutions by using a 2D multipole representation in the form of a Laurent series, which enables us to find static equilibria. For this general kind of a conformal mapping, we exclude the region around the singularity $(x, y)=(0,0)$ with $\varrho=\sqrt{x^{2}+y^{2}}<R_{\mathrm{ts}}$ within the termination shock, representing the inner part of the astrosphere. We define $u:=x+\mathrm{i} y$. $\mathcal{A}$ and $\mathcal{B}$ are the complex magnetic flux function and the complex magnetic field. $\mathcal{A}(u)$ and $\mathcal{B}(u)$ are holomorphic functions (at least nearly everywhere), with a real part $\mathfrak{R}(\mathcal{A})=\phi_{m}$ and imaginary part $\mathfrak{I}(\mathcal{A})=A$, and $\mathfrak{R}(\mathcal{B})=B_{x}$ and $\mathfrak{J}(\mathcal{B})=-B_{y}$ accordingly. $A$ is the magnetic flux function and $\phi_{m}$ is the scalar magnetic potential. Therefore, we can write

$\mathcal{B}=\frac{\mathrm{d} \mathcal{A}}{\mathrm{d} u}=\frac{\partial \phi_{m}}{\partial x}+\mathrm{i} \frac{\partial A}{\partial x}=B_{x}-\mathrm{i} B_{y}$.

We use the following Ansatz for the magnetic field

$\mathcal{B}=B_{S \infty}+\sum_{\mu=1}^{\infty} c_{\mu} u^{-\mu}$

to satisfy the asymptotical boundary conditions

$\lim _{|u| \rightarrow \infty} \mathcal{B}=B_{S \infty}$

so

$\mathcal{A}=B_{S \infty} u+C_{0} \ln u+\sum_{v=1}^{\infty} C_{\nu} u^{-v}$

is valid.

There is a similarity between the logarithmic term of the hydrodynamical problem of a circular flow and the radial or azimuthal part of a magnetic potential field, so that we can write

$\mathcal{A}=B_{S \infty} u+\frac{\Gamma}{2 \pi i} \ln u+\sum_{v=1}^{\infty} C_{v} u^{-v}$,

or especially

$\mathcal{A}=B_{S \infty} u+\frac{\Gamma_{0}\left(\cos \beta_{0}+\mathrm{i} \sin \beta_{0}\right)}{2 \pi \mathrm{i}} \ln u-\left|C_{1}\right| \frac{\left(\cos \beta_{1}+\mathrm{i} \sin \beta_{1}\right)}{u}$

$-\left|C_{2}\right| \frac{\left(\cos \beta_{2}+\mathrm{i} \sin \beta_{2}\right)}{u^{2}}+$ terms of higher order.

The first term in the expansion is the homogenous part due to the asymptotical boundary condition, which survives the noncanonical transformation. The second term is the circulation or monopole part due to a line current. If $\Gamma=\Gamma_{0}\left(\cos \beta_{0}+\mathrm{i} \sin \beta_{0}\right)$ is real, then one has a typical counterflow configuration for the hydrodynamcal circulation of a flow around a circular cylinder. Here $\Gamma_{0}=\mu_{0} I_{0}$, where $I_{0}$ is the line current. The third term 
is a line-dipole part. In the case of pure hydrodynamics, this part represents the radius $R^{2} \equiv\left|C_{1}\right|$ of a flow around a circular cylinder, if $\sin \beta_{1}=0$. The fourth term is the quadrupole part. Furthermore, $\left|C_{1}\right|=2 I_{2} a$ is the dipole moment, $I_{2}$ the current, $a$ the half distance of the antiparallel line currents, and the product $I_{2} a=$ const, while $I_{2} \rightarrow \infty$ and $a \rightarrow 0$.

Moving stars, together with their winds, can be regarded as obstacles in the stream of the ISM. Such counterflow configurations lead to the formation of separating surfaces. On these surfaces, stagnation points must exist where the flow velocity vanishes. Stream lines passing through these stagnation points are called separatrices because they separate stream lines of different topological connection. They represent the borderlines between two different flows. To calculate the stagnation points ${ }^{3}$, one has to solve the equation $\mathcal{B}=0$. In the topological theory of fluids, this field structure is called an X-point structure ${ }^{4}$. However, application to the Parker field would imply the existence of an additional singularity beyond the X-point, located at the origin of the magnetized wind plasma, i.e. at the location of the central star itself. To generate a field similar to the hydrodynamic Parker flow of the heliospheric flow field (Parker 1961), it is necessary (i) that $x_{S P} \equiv x_{N}(<0)$ is the magnetic neutral point (where the magnetic field vanishes and in our treatment also the velocity field), (ii) that for $\varrho \equiv \sqrt{\left(x^{2}+y^{2}\right)} \rightarrow 0$ a radial field structure exists, and (iii) that the flow field converges asymptotically to a homogenous field for $\varrho \rightarrow \infty$. The Parker flow field is only useful and valid far away from the origin of the magnetized wind plasma of the central star. One reason is, of course, that the field strength towards the origin is diverging. It turns out that selection of a circulation with a nonvanishing imaginary part is necessary. This enables the stellar wind to escape from the region of the reverse shock, and it creates a radial field structure towards the origin.

Note: in our case, the Alfvén or the usual Mach number does not determine the geometrical shape of the astropause, in contrast to the discussion in Parker (1961). Hence, our method cannot be compared directly with Parker's calculations. The mapping technique will allow sub- and super-Alfvenic flows to exist, although the streamline geometry does not change. Thus, it is also possible to have open field lines on both sides (upwind and downwind), like in the purely magnetic model of Parker.

\subsection{Neutral points and multipole moments}

The complex magnetic field $\mathcal{B}$ can be calculated from $\mathcal{A}=\phi_{m}+$ $\mathrm{i} A$, with $\phi_{m}$ as the magnetic potential

$\mathcal{B}=\frac{\mathrm{d} \mathcal{A}}{\mathrm{d} u}$

where the Ansatz of the series (56) yields

$\mathcal{B}=B_{S \infty}+\frac{\Gamma}{2 \pi \mathrm{i}} \frac{1}{u}-\frac{C_{1}}{u^{2}}-\frac{2 C_{2}}{u^{3}}$.

A direct analytical method of calculating the null points should be applied here in the case of a multipole representation with two non vanishing multipoles. We restrict our analysis to the first two multipoles to get the magnetic null:

$\mathcal{B}=B_{S \infty}+\frac{\Gamma}{2 \pi \mathrm{i}} \frac{1}{u}-\frac{C_{1}}{u^{2}}=0$.

${ }^{3}$ For a regular configuration, in the sense of finite Mach number and density distribution, these nulls or magnetic neutral points are also stagnation points.

${ }^{4}$ Such points are saddle points of the magnetic flux function.
This gives a quadratic equation,

$u^{2}-\frac{\mathrm{i} \Gamma}{2 \pi B_{S \infty}} u-\frac{C_{1}}{B_{S \infty}}=0$,

having the solutions

$$
\begin{aligned}
u & =\frac{\mathrm{i} \Gamma}{4 \pi B_{S \infty}} \pm \sqrt{\left(\frac{\mathrm{i} \Gamma}{4 \pi B_{S \infty}}\right)^{2}+\frac{C_{1}}{B_{S \infty}}} \\
& =\frac{\mathrm{i}\left(\Gamma_{\mathrm{r}}+\mathrm{i} \Gamma_{\mathrm{i}}\right)}{4 \pi B_{S \infty}} \pm \sqrt{\left(\frac{C_{1 \mathrm{r}}}{B_{S \infty}}+\frac{\Gamma_{\mathrm{i}}^{2}-\Gamma_{\mathrm{r}}^{2}}{16 \pi^{2} B_{S \infty}^{2}}\right)+\mathrm{i}\left(\frac{C_{1 \mathrm{i}}}{B_{S \infty}}-\frac{\Gamma_{\mathrm{r}} \Gamma_{\mathrm{i}}}{8 \pi^{2} B_{S \infty}^{2}}\right)} \\
& =\frac{-\Gamma_{\mathrm{i}}+\mathrm{i} \Gamma_{\mathrm{r}}}{4 \pi B_{S \infty}} \pm \sqrt{\frac{\sqrt{\mathcal{R}^{2}+\mathcal{I}^{2}}+\mathcal{R}}{2}} \pm \frac{\mathrm{i} \mathcal{I}}{\sqrt{2 \sqrt{\mathcal{R}^{2}+\mathcal{I}^{2}}+2 \mathcal{R}}},
\end{aligned}
$$

with

$\mathcal{R}=\frac{C_{1 \mathrm{r}}}{B_{S \infty}}+\frac{\Gamma_{\mathrm{i}}^{2}-\Gamma_{\mathrm{r}}^{2}}{16 \pi^{2} B_{S \infty}^{2}}, \quad \mathcal{I}=\frac{C_{1 \mathrm{i}}}{B_{S \infty}}-\frac{\Gamma_{\mathrm{r}} \Gamma_{\mathrm{i}}}{8 \pi^{2} B_{S \infty}^{2}}$,

where the indices $r$ and $i$ indicate the real and imaginary parts of the coefficients. Therefore,

$x_{S 1}=-\frac{\Gamma_{\mathrm{i}}}{4 \pi B_{S \infty}} \pm \frac{1}{\sqrt{2}}\left(\sqrt{\sqrt{\mathcal{R}^{2}+\mathcal{I}^{2}}+\mathcal{R}}\right)$
$y_{S 1}=\frac{\Gamma_{\mathrm{r}}}{4 \pi B_{S \infty}} \pm \frac{I}{\sqrt{2 \sqrt{\mathcal{R}^{2}+\mathcal{I}^{2}}+2 \mathcal{R}}}$

The singular point is situated where field lines meet; here is the beginning, i.e. the ending of several field lines. The stagnation point also marks one contour, e.g. a certain value of $A$. From that, we can calculate the equation of the astropause and the asymptotical equation of the astropause, which delivers the diameter of the astrotail at infinity. The second stagnation point, which is calculated for the case of the symmetric linedipole with $y_{S 2}=0$, $x_{S 2}>0>x_{S 1}$, and $x_{S 2}>\left|x_{S 1}\right|$, can be considered as the radius of the inner astrosphere, i.e. as the position of the termination shock in the direction of the astrotail. We perform a systematic calculation of the magnetic neutral points, making the general Ansatz of a Laurent series. If the Alfvén Mach number is finite, the magnetic neutral points are identical with the stagnation points. We analyse how the position of the stagnation points of the interstellar counterflow influences the field structure, especially the geometry of the astropause. We use the logarithmic part and the homogeneous asymptotic boundary condition, together with the assumption of a finite number of neutral points:

$\mathcal{A}=B_{S \infty} u+C_{0} \ln u+\sum_{v=1}^{N} C_{v} u^{-v}$.

Here $v=1$ is the dipole, $v=2$ the quadrupole, $v=3$ the octopole, etc. The magnetic null or neutral points $u_{k}$ are given by

$\mathcal{B}\left(u_{k}\right)=\left.\frac{\mathrm{d} \mathcal{A}}{\mathrm{d} u}\right|_{u=u_{k}}=0$.

Thus, we have to find the null points of the polynomial

$u^{N+1}+\frac{C_{0}}{B_{S \infty}} u^{N}-\sum_{v=1}^{N} \frac{v C_{v}}{B_{S \infty}} u^{N-v}=\prod_{k=1}^{N+1}\left(u-u_{k}\right)=0$. 
With the help of Vieta's theorem of roots, we get

$C_{0}=-B_{S \infty} \sum_{k=1}^{N+1} u_{k}$

$C_{v}=(-1)^{v} \frac{B_{S \infty}}{v} \sum_{\cup C_{v+1}^{N+1}}\left(\prod_{u_{k} \in C_{v+1}^{N+1}} u_{k}\right), 1 \leq v \leq N$.

The symbol $C_{v+1}^{N+1}$ denotes combinations of the $u_{k}$. These are the subset $v+1$ elements of the $N+1$ elements (of the magnetic nulls). The distribution of the magnetic neutral points determines the global geometry and the topology of an astrosphere.

\subsection{The equation of the astropause}

Regarding a symmetric and, with respect to the direction of interstellar medium, closed astrosphere, we only take neutral points on the $x$ axis into account. The smallest, negative, $x$ value gives

$A\left(u_{1}\right)=A_{\text {sep } 1}=A\left(x_{1}, 0\right) \quad \Rightarrow \quad A_{\text {sep } 1}=0$,

and, therefore,

$A(x, y)=A_{\text {seplu }}=0$

for the separatrix with $x<0$. However, including the monopole term, we obtain for the point where the separatrix (astropause) passes through the $y$ axis:

$\lim _{x \rightarrow-0} A(x, y)=A\left(x=-0, y=y_{D}\right)=A_{\text {sep } 1}$.

where $y_{D}$ is the location of the astropause, which is lying on the $y$ axis and, therefore, is elongated parallel to the inner astrosphere; i.e. it is positioned at the same $x$ coordinate of the star $\left(x_{\text {star }}, y_{\text {star }}\right)=(0,0)$. Now, $y_{D}$ can be determined from Eq. $(71)$. For $x>0$, we may calculate the curve of the astropause in the $x-y$ plane as an implicit function of

$$
\begin{aligned}
A_{H}(x, y) & =A\left(x=-0, y=y_{D}\right)+\pi C_{0} \\
& =A_{\text {sep } 1}+\pi C_{0}=A_{\text {sep } 2}=\pi C_{0},
\end{aligned}
$$

taking the jump of the arcus tangens function into consideration. With three neutral points, the magnetic flux function can be written as

$$
\begin{aligned}
\mathfrak{J}(\mathcal{A})=A= & B_{S \infty} y+C_{0} \arctan \left(\frac{y}{x}\right) \\
& -C_{1} \frac{y}{x^{2}+y^{2}}-C_{2} \frac{2 x y}{\left(x^{2}+y^{2}\right)^{2}} .
\end{aligned}
$$

For $x<0$ and $y>0$ (with opposite sign for the limit of the $\arctan$ for $y<0$, i.e. for the down part of the astropause),

$\lim _{x \rightarrow-0} A(x, y)=A\left(0, y_{D}\right)=B_{S \infty} y_{D}-\frac{\pi}{2} C_{0}-\frac{C_{1}}{y_{D}}=0=A_{\text {sep } 1}$,

we get for the point where the separatrix intersects the $y$ axis

$y_{D}= \pm\left(\frac{\pi C_{0}}{4 B_{S \infty}}+\sqrt{\left(\frac{\pi C_{0}}{4 B_{S \infty}}\right)^{2}+\frac{C_{1}}{B_{S \infty}}}\right)$.

In the case of general asymmetric configurations, it is possible to calculate the intersection point with the $y$ axis from

$$
\begin{aligned}
\lim _{x \rightarrow-0} A\left(x, y_{D}\right) & =A\left(x_{1}, y_{1}\right)=A_{\text {sep } 1} \\
& =B_{S \infty} y_{D}-\frac{\pi}{2} C_{0 \mathrm{r}}+C_{0 \mathrm{i}} \ln \left|y_{D}\right|+\sum_{v=1}^{N} C_{v} y_{D}^{-v} .
\end{aligned}
$$

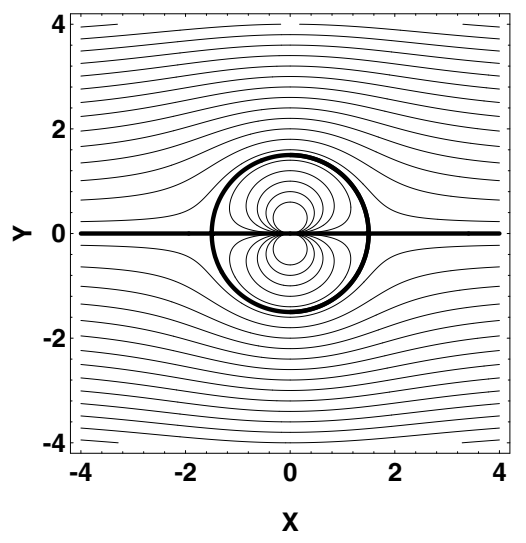

Fig. 2. Field lines of the tail model as contour lines of the magnetic flux function and branches of the separatrix. $u_{1}$ is fixed at -1.5 , where the scale is in units of $100 \mathrm{AU}$. For $u_{2}=1.5$ one can clearly see the similarity of this magnetic field lines with that of a flow around a cylinder.

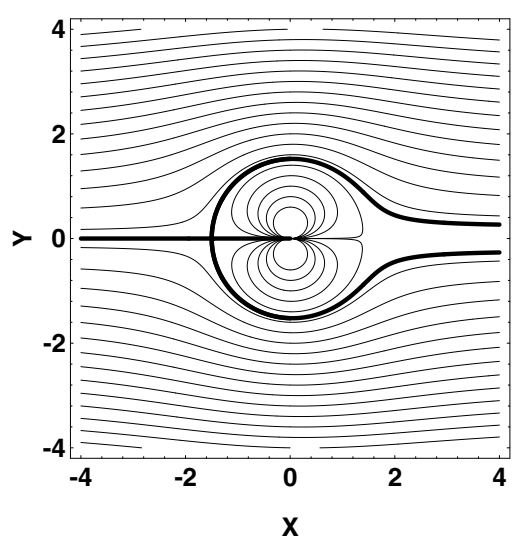

Fig. 3. As in Fig. 2, but for $u_{2}=0.95 \times 1.5=1.425$.

With

$\lim _{x \rightarrow+0} A\left(x, y_{D}\right)=B_{S \infty} y_{D}+C_{0} \frac{\pi}{2}-\frac{C_{1}}{y_{D}}=A_{\mathrm{sep} 1}+\pi C_{0}=\pi C_{0}$,

we can calculate the other branch of the separatrix:

$$
\begin{aligned}
A_{H}(x, y) & =B_{S \infty} y_{D}+\frac{\pi}{2} C_{0 \mathrm{r}}+C_{0 \mathrm{i}} \ln \left|y_{D}\right|+\sum_{v=1}^{N} C_{v} y_{D}^{-v} \\
\stackrel{\text { here }}{=} & B_{S \infty} y+C_{0} \arctan \left(\frac{y}{x}\right)-C_{1} \frac{y}{x^{2}+y^{2}}-C_{2} \frac{2 x y}{\left(x^{2}+y^{2}\right)^{2}} \\
& =\pi C_{0},
\end{aligned}
$$

which is the part of the astropause that is opened in the tail direction. The outer separatrix is nothing else than the astropause. The asymptotical equation of the astropause is given by

$$
\begin{aligned}
A_{H \infty}(x, y) & :=\lim _{x \rightarrow \infty} A\left(x, y_{H}\right)=B_{S \infty} y_{H} \\
& =\pi C_{0} \Rightarrow y_{H}=\frac{\pi C_{0}}{B_{S \infty}} .
\end{aligned}
$$

In the series of Figs. 2 to 9 we show how the existence of two null points influences the shape of the astropause as an astrospheric interface. The scale is in units of $100 \mathrm{AU}$. The first neutral point is chosen at a location that results in an astropause configuration applicable to the heliosphere. 


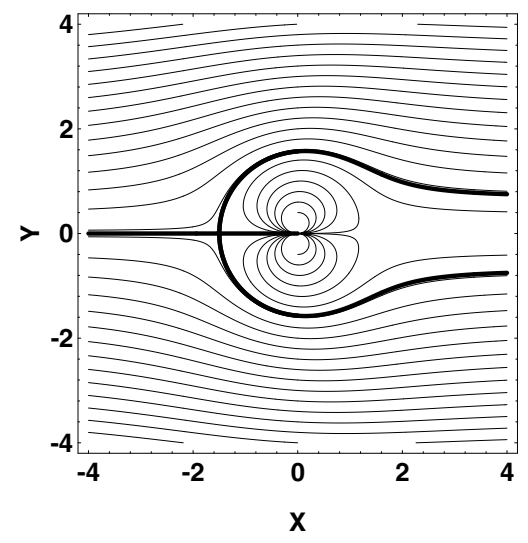

Fig. 4. As in Fig. 2, but for $u_{2}=0.85 \times 1.5=1.275$. That some field lines do not appear closed is only a plotting artifact.

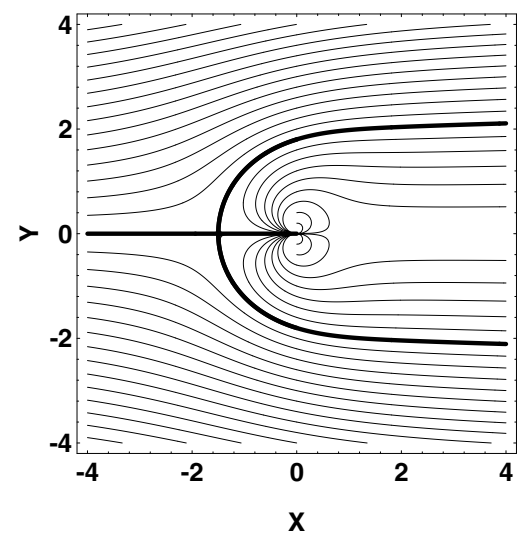

Fig. 5. As in Fig. 2, but for $u_{2}=0.5 \times 1.5=0.75$. That some field lines do not appear closed is only a plotting artifact.

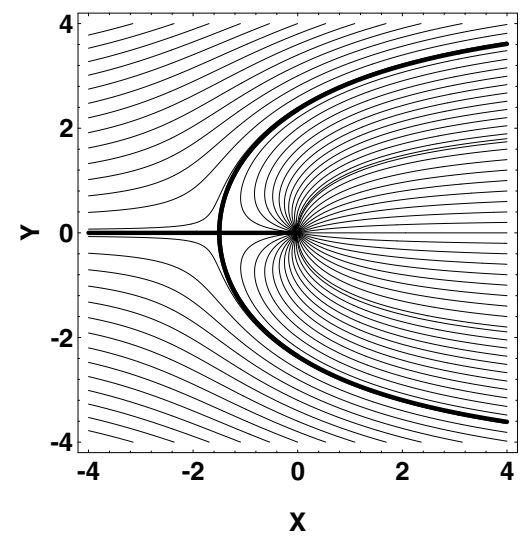

Fig. 6. As in Fig. 2, but for $u_{2}=0$. In this special case the tail is similar to the Parker model (1961). $u_{2}=0$ is not a null point, because this is the position of the singularity. $u_{2}=0$ is only due to the fact that in the sum of Eq. (64) only a monopole moment appears; see Eq. (68).

If we fix the neutral point, $x_{1}$, in front of the astrosphere, we see that for $x_{2}=-x_{1}=R$ the fieldline geometry looks like that of a hydrodynamical counterflow of a cylinder with radius $R$ (Fig. 2). By displacing the second null point towards the origin, i.e. for $0<x_{2}<-x_{1}=R$, the separatrix breaks up, and a taillike channel is formed causing the drop-like shape of the astropause. This tail opens up for a null point approaching the origin (Figs. 3-5). For $x_{2}=0$, the astrosphere has a typical Parker shape (Fig.6). Further displacement to negative values of $x_{2}$

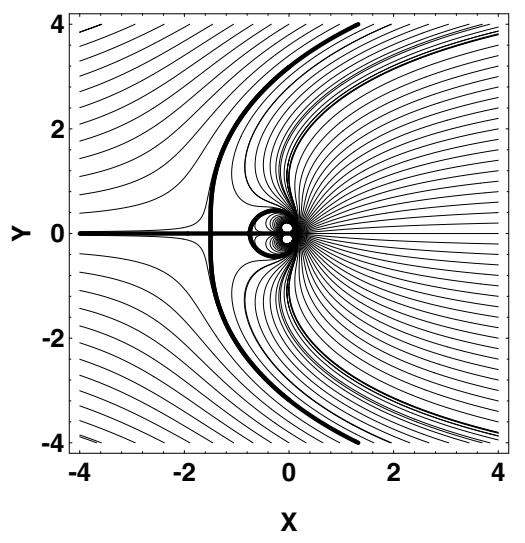

Fig. 7. As in Fig. 2, but for $u_{2}=-0.5 \times 1.5=-0.75$. Here, one gets a region with closed field lines in the downstream direction.

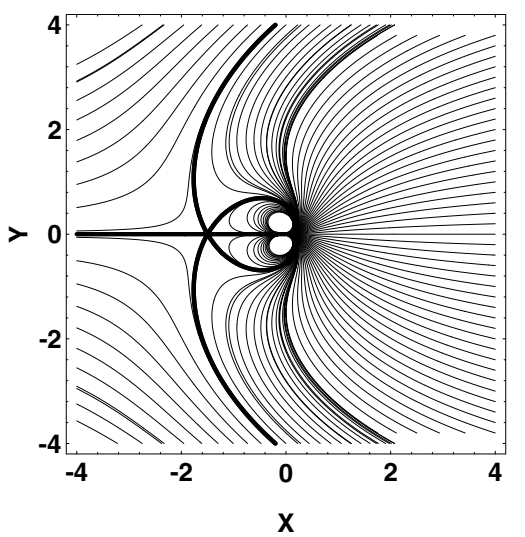

Fig. 8. As in Fig. 2, but for $u_{2}=-1.5$; here we have a neutral point of second order.

results in the formation of an anti-tailward bubble within the actual astropause (Fig. 7), leading to a notch in the astropause nose. This bubble grows until it touches the first neutral point resulting in a neutral point of second order (Fig. 8). Here, the absolute value of the monopole moment is highest ${ }^{5}$. A completely different scenario is shown in Fig. 9. Here, we have only one null point off the $x$-axis. This implies a complex circulation $\Gamma$, leading to a spiral structure in the vicinity of the origin and emulating the Parker spiral. This represents an inner boundary condition giving a strong azimuthal component (a strong winding) of the magnetic field in the equatorial plane of the heliosphere.

\subsection{Discussion of the mirror symmetric case}

In the case of $\Gamma_{\mathrm{r}}=0$ and $C_{1 i}=0$, we get an equilibrium that is mirror symmetric with respect to the $x$ axis. The magnetic null points read

$u=\frac{-\Gamma_{\mathrm{i}}}{4 \pi B_{S \infty}} \pm \sqrt{\frac{C_{1 \mathrm{r}}}{B_{S \infty}}+\frac{\Gamma_{\mathrm{i}}^{2}}{16 \pi^{2} B_{S \infty}^{2}}}$.

5 Also that of the dipole moment. The fieldlines of that dipole escape to the right if the second null point is shifted from the right to the left of the origin, so that the radial "outflow" is stronger compared to the other images. The displacement of the second neutral point influences the inner boundary conditions for the field lines. 


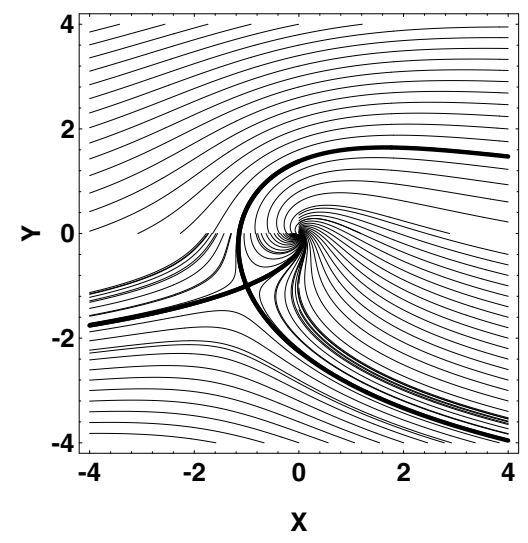

Fig. 9. Field lines of the tail model as contour lines of the magnetic flux function and separatrices for $u_{1}=-1.0-i=(x, y)=(-1,-1)$; this is a potential field with a real valued circulation that shows the existence of a spiral-shaped field structure in the inner heliosphere, i.e. an azimuthal component; the thus imitated Parker spiral should extend into the outer heliosphere. Due to a plotting artifact, not all calculated field lines are complete.

One can easily see that, if

$\frac{C_{1 \mathrm{r}}}{B_{S \infty}}+\frac{\Gamma_{\mathrm{i}}^{2}}{16 \pi^{2} B_{S \infty}^{2}}<0 \quad \Longleftrightarrow \quad C_{1 \mathrm{r}}<-\frac{\Gamma_{\mathrm{i}}^{2}}{16 \pi^{2} B_{S \infty}}$,

two magnetic nulls occur, which are not lying on the $x$ axis, and the astrosphere is open with respect to the counterflow direction. For

$C_{1 \mathrm{r}}=-\frac{\Gamma_{\mathrm{i}}^{2}}{16 \pi^{2} B_{S \infty}}$

only one stagnation point exists. The second null, $u=0$, is not a real null point, but a pole. The first stagnation point is far away from the origin (i.e. from the star). The dipole part of the outflow, or the magnetic field, is like the resistance of a flow around an obstacle. Under the assumption $C_{1 \mathrm{r}}=B_{S \infty} R^{2}$, we get

$u=x=\frac{-\Gamma_{\mathrm{i}}}{4 \pi B_{S \infty}} \pm R \sqrt{1+\frac{\Gamma_{\mathrm{i}}^{2}}{16 \pi^{2} B_{S \infty}^{2} R^{2}}}$.

For $\Gamma_{\mathrm{i}} \rightarrow 0$, we get two stagnation points $(x=+R$ and $x=-R)$, positioned on a circle with radius $R$. Figure 2 shows that the fieldlines of this magnetic field are identical to the image of streamlines of a flow around a body shaped like a circular cylinder (keeping in mind the substitution $\phi_{m} \rightarrow \phi, A \rightarrow \psi$, with $\mathcal{W}=\phi+\mathrm{i} \psi$ as the hydrodynamical potential and $\left.B_{S_{\infty}} \rightarrow v_{\infty}\right)$. The circle is a separatrix that separates fieldlines of different topology. This astrosphere, however, would have the disadvantage of being a closed surface (line in 2D), and there would be no possibility that plasma could flow into the tail. This would imply a diffusion process, as is described in Neutsch \& Fahr (1983).

\section{Pure sub- or super-Alfvénic flows}

A tangential discontinuity has to form due to magnetic shear, Here two different, magnetized plasmas encounter. At least, one has to expect a strong gradient perpendicular to the magnetic field involving a non-singular current sheet. For typical astrophysical plasmas, the structure of such a current sheet can be derived by solving the coupled system of Vlasov and Maxwell equations selfconsistently, assuming the symmetry of the previous section. This kind of translation invariant plasma, although collisionless, can be considered to follow a quasiMaxwellian distribution function, so that we can use the solution of Harris (1962). Such a current sheet can be seen as the prototype of a current sheet separating two plasmas. To enable a constant asymptotic homogenous field and to mimic two astropause current sheet positions, where the two symmetric branches of the potential field separatrices should be localized, we choose the following transformation equation

$$
\begin{aligned}
\alpha(A)= & \frac{\operatorname{sign}\left[\alpha^{\prime}\right] \mathrm{A}}{\sqrt{1-M_{\mathrm{A} \infty}^{2}}}+a_{1} \ln \cosh \frac{\frac{A}{B_{s \infty}}-y_{1}}{d_{1}} \\
& +a_{2} \ln \cosh \frac{\frac{A}{B_{s \infty}}-y_{2}}{d_{2}},
\end{aligned}
$$

with derivative

$$
\begin{aligned}
\alpha^{\prime}(A)= & \frac{\operatorname{sign}\left[\alpha^{\prime}\right]}{\sqrt{1-M_{\mathrm{A} \infty}^{2}}}+\frac{a_{1}}{B_{S \infty} d_{1}} \tanh \frac{\frac{A}{B_{s \infty}}-y_{1}}{d_{1}} \\
& +\frac{a_{2}}{B_{S \infty} d_{2}} \tanh \frac{\frac{A}{B_{s \infty}}-y_{2}}{d_{2}} \\
= & \frac{B_{\infty}}{B_{S \infty}}+\frac{a_{1}}{B_{S \infty} d_{1}} \tanh \frac{\frac{A}{B_{s \infty}}-y_{1}}{d_{1}} \\
& +\frac{a_{2}}{B_{S \infty} d_{2}} \tanh \frac{\frac{A}{B_{s \infty}}-y_{2}}{d_{2}}
\end{aligned}
$$

where $\operatorname{sign}\left[\alpha^{\prime}\right]$ indicates that the asymptotical magnetic field can be parallel or anti-parallel to the asymptotical flow. Hence, with Eq. (86),

$\operatorname{sign}\left[\alpha^{\prime}\right] \equiv \operatorname{sign}\left[B_{\infty}\right]$.

This transformation fulfills the symmetric asymptotic boundary conditions for the magnetic field, given by

$\lim _{y \rightarrow \infty} \boldsymbol{B}=B_{\infty} \boldsymbol{e}_{x}=\lim _{y \rightarrow-\infty} \boldsymbol{B}$,

where $B_{\infty}$ is a constant. In the case of an MHS equilibrium, where

$A=B_{S \infty} y \quad$ with $\quad B_{S \infty}>0$,

the second term and the third term of Eq. (85), which represent the outer current sheets, are concentrated around $y_{1}$ and $y_{2}$, which should be the locations of the astropause envelope borders in 2D (asymptotically). This implies that $\alpha^{\prime}$ is the amplification factor of the static magnetic field

$\boldsymbol{B}=\boldsymbol{\nabla} \alpha \times \boldsymbol{e}_{z}=\alpha^{\prime} \boldsymbol{\nabla} A \times \boldsymbol{e}_{z}=\alpha^{\prime} B_{s \infty} \boldsymbol{e}_{x}$,

which shows the behaviour of the asymptotic magnetic field if the magnetohydrostatic field is homogenous. The stationary field at infinity $(x \rightarrow \infty)$ can only depend on $y$ because $A$ converges to a value proportional to $y$ and, therefore, $\alpha(A) \sim \alpha(y)$. The symmetric boundary condition Eq. (88) leads then to

$\frac{a_{1}}{d_{1}}=-\frac{a_{2}}{d_{2}}=: B_{1}$, 
which means that the current sheets have different signs (antiparallel currents). The sign of the Jacobian (here $\alpha^{\prime}$ ) must be unique in the whole domain, as there should be no roots for a purely sub-Alfvénic or super-Alfvénic flow. In addition, we make the assumption that $0<d_{1}=d_{2} \ll\left|y_{1}-y_{2}\right|$ and $y_{1}>y_{2}$, $y_{1}=-y_{2}>0$ for the symmetric case (to ensure a highly symmetric equilibrium).

\subsection{Examples of noncanonical transformations for sub-Alfvénic flows}

With the above assumptions, Eqs. (86) and (91), we make certain that

$\left|\frac{\mathrm{d} \alpha}{\mathrm{d} A}\right|=\left|\alpha^{\prime}\right|>1$,

so that the flow is sub-Alfvénic. With condition (91), (92) can be written as

$\left|\alpha^{\prime}(y=0)\right|=\left|\frac{\operatorname{sign}\left[\alpha^{\prime}\right]}{\sqrt{1-M_{\mathrm{A} \infty}^{2}}}-\frac{2 B_{1}}{B_{S \infty}}\right|=\left|\frac{B_{\infty}}{B_{S \infty}}-\frac{2 B_{1}}{B_{S \infty}}\right|>1$,

with

$\frac{B_{\infty}}{B_{S \infty}}=\frac{\operatorname{sign}\left[\alpha^{\prime}\right]}{\sqrt{1-M_{\mathrm{A} \infty}^{2}}} \cdot$

This leads to the following restrictions

For $B_{\infty}>0 \Leftrightarrow \operatorname{sign}\left[\alpha^{\prime}\right]>0$

$$
\begin{array}{r}
2 B_{1}<B_{\infty}\left(1-\sqrt{1-M_{\mathrm{A} \infty}^{2}}\right) \\
\vee \quad 2 B_{1}>B_{\infty}\left(1+\sqrt{1-M_{\mathrm{A} \infty}^{2}}\right),
\end{array}
$$

for $B_{\infty}<0 \Leftrightarrow \operatorname{sign}\left[\alpha^{\prime}\right]<0$

$$
\begin{aligned}
2 B_{1}<B_{\infty}\left(1+\sqrt{1-M_{\mathrm{A} \infty}^{2}}\right) \\
\vee \quad 2 B_{1}>B_{\infty}\left(1-\sqrt{1-M_{\mathrm{A} \infty}^{2}}\right) .
\end{aligned}
$$

The amplification factor $\alpha^{\prime}$ of the static asymptotic magnetic field $B_{s \infty}$ at the axis of symmetry tells us that a magnetic jump occurs at the location of the separatrix, because the magnetic fields from the inside and outside converge to different values. The strength of the jump is given by

$2 B_{1}=B_{\infty}-\alpha^{\prime}(y=0) B_{S \infty}=B_{\infty}\left(1-\frac{\sqrt{1-M_{\mathrm{A} \infty}^{2}}}{\sqrt{1-M_{\mathrm{A}, i}^{2}}}\right)$.

Therefore, not all values of the inside magnetic field are allowed if the flow is purely sub-Alfvénic. The inside magnetic field $B_{i}$ is given by

$B_{\mathrm{i}}=B_{\infty}-2 B_{1}=B_{\infty} \frac{\sqrt{1-M_{\mathrm{A} \infty}^{2}}}{\sqrt{1-M_{\mathrm{A}, \mathrm{i}}^{2}}}$.

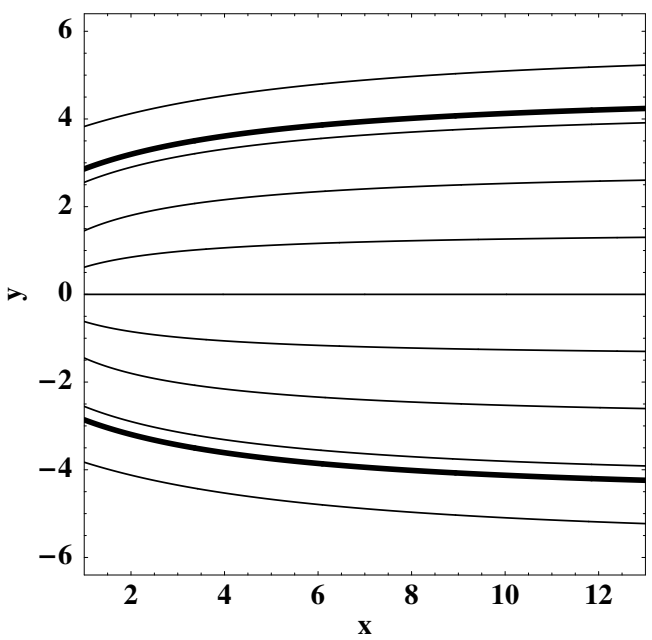

Fig. 10. Regarding the field lines and especially the separatrix (thick line) one can see that they are not identical with the isocontours of the electric current plotted in Fig. 11.

Therefore, the polarity cannot change from the outside to the inside. With Eqs. (100) and (93), we get a lower limit for the inside magnetic field if the outside magnetic field $B_{\infty}$ and the outside asymptotical Alfvén Mach number $M_{\mathrm{A} \infty}$ are given:

$\left|B_{\mathrm{i}}\right|>\sqrt{1-M_{\mathrm{A} \infty}^{2}}\left|B_{\infty}\right|=B_{S \infty}$.

Boundary conditions for the 1D case are given by the condition Eq. (100). This implies that the outer and inner Alfvén Mach numbers are the boundary conditions for Eq. (14), together with the choice $B_{\infty}=$ const. This determines $B_{\mathrm{i}}$, which cannot be prescribed as a boundary condition, since the problem would be overdetermined. With the above relations we see that the possibility of setting boundary conditions is reduced, due to the reflection symmetry condition for the magnetic field. We choose the axis of symmetry in the $x-y$ plane, the $x$-axis as second boundary, and demand only regularity on the other two boundaries $x=1$ and $x=x_{\text {tail-end }}$. Regularity is guaranteed by the behaviour of the potential field and the transformation type in this domain.

We can also use the above transformation to fulfill the boundary condition for the asymptotical $1 D$ region of a $2 D$ field. In this case, the boundary conditions are mapped together with the mapping of the whole 2D potential field because we know in advance that this will again be a stationary equilibrium state with field-aligned incompressible flow. Therefore, if for $x \rightarrow \infty$, the equilibrium converges asymptotically to the $1 \mathrm{D}$ equilibria given by Eqs. (14) and (86), this method can be used. We can take any of the given potential fields in the foregoing section, if we want to keep the potential character of the magnetic field in the tail. This leads then to a 2D sub-Alfvénic equilibrium state, writing

$\lim _{x \rightarrow \infty} \alpha(A(x, y))=\alpha\left(A_{\infty}\right), \quad$ with $\quad A_{\infty}=B_{S \infty} y$.

In Figs. 10 and 11, where we plotted the field lines and separatrix and the isocontours of the current density, an interesting feature of the transformation can be seen: while in magnetohydrostatics, where $\boldsymbol{B} \cdot \boldsymbol{\nabla} P=0$ and $\boldsymbol{j} \cdot \boldsymbol{\nabla} P=0$ imply that the current is constant on field lines, so that the current isocontours coincide with the magnetic field lines, the situation is now completely different. Comparing Fig. 11 with the fieldlines in Fig. 10, it can be seen that, asymptotically, the field lines and isocontours of the current density geometrically converge, but topologically 


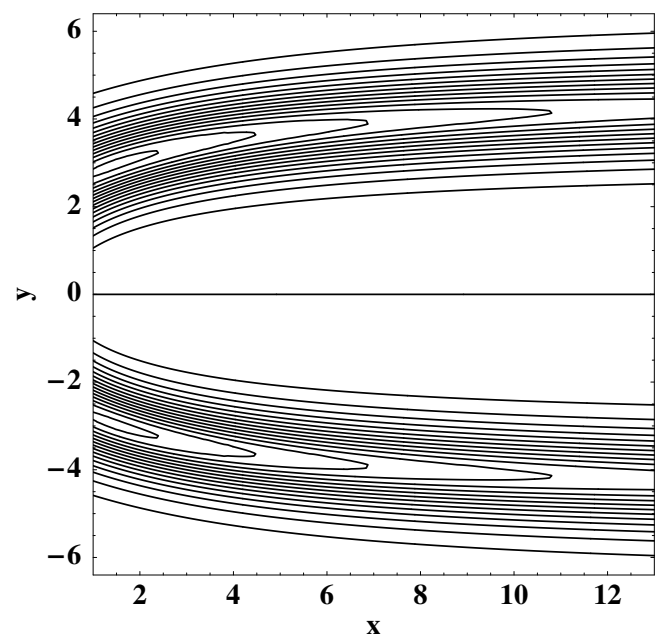

Fig. 11. Isocontours of the current density, where the high current isocontours are obviously concentrated around the heliopause. The curvature and closure of the current isocontours can be seen clearly in contrast to the curved, but open separatrix line in Fig. (10); only in the asymptotical 1D region, i.e. for $x \rightarrow \infty$, the lines of maximum current and the separatrix (astropause) shapes seem to converge.

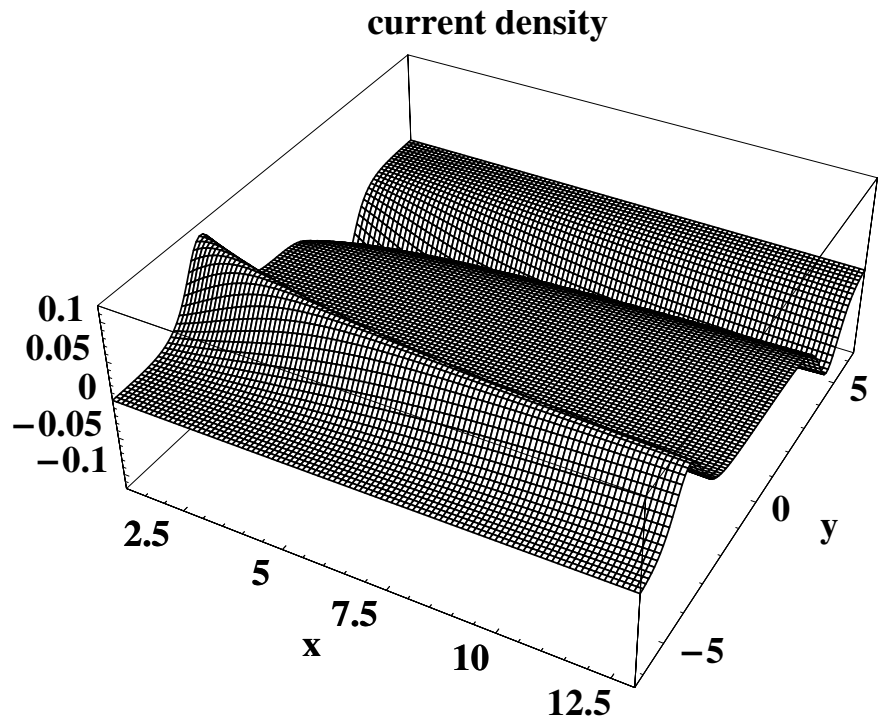

Fig. 12. Shown is the current density and its increase towards the inner astrosphere, i.e. the downwind region of the reverse shock. Also visible are current sheets with a width of $100 \mathrm{AU}$.

they are different: while the field lines are open throughout the tail, the isolines of the current are closing in the vicinity of the separatrix (i.e. the astropause).

To clearly show these differences in the field lines (Fig. 10) and the isocontours of the current density (Fig. 11), a finite width of the current sheet of $100 \mathrm{AU}$ has been used. These broad current sheets are visible in Fig. 12 where we have plotted the strength of the current density, whose absolute value increases towards the termination shock region.

We now turn to the presentation of an example of a toy model that might represent the heliospheric tail region. First, we reduce the width of the current sheet to a more realistic value. That thickness can be estimated from the fact that it should be larger than several ion gyroradii (Neutsch \& Fahr 1983; Fahr et al. 1982). The ion gyroradius is at least of the order of $10^{2}-10^{3} \mathrm{~km}$, and we set the width of the current sheet to $10 \mathrm{AU}$.

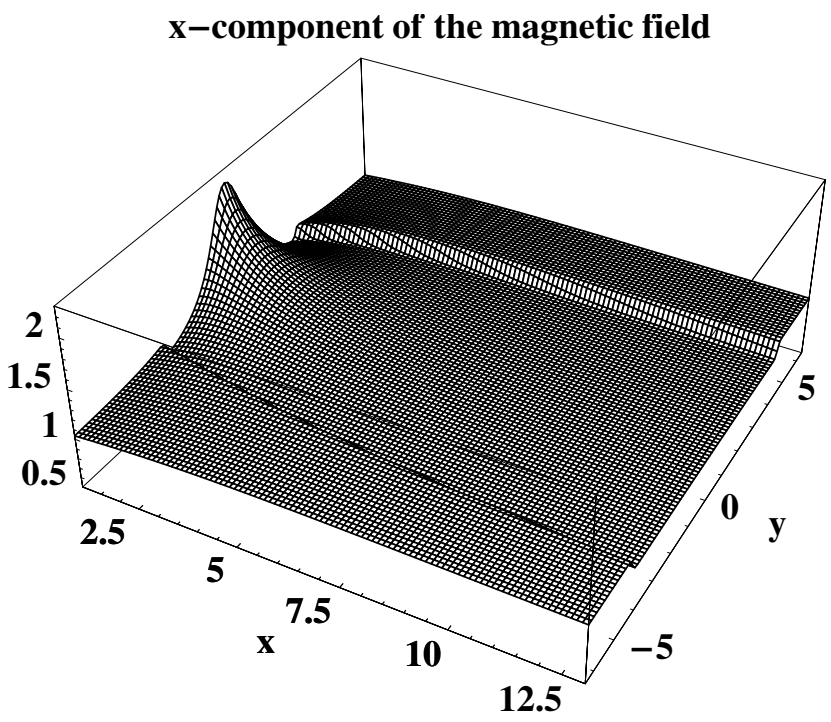

Fig. 13. The $x$-component of the magnetic field. It rises towards the location of the termination shock caused by the magnetic monopole. Clearly visible are the jumps at the locations of the current sheets.

In addition, we can fall back on the measurements and estimated values given in Frisch et al. (2004) (Table 1, Model 2) ${ }^{6}$. For the outside magnetic field we take $B_{\infty}=5 \mu \mathrm{G}$, for the proton density $n_{\mathrm{i}} \approx n_{\mathrm{e}}=0.1 \mathrm{~cm}^{-3}$ and for the velocity $v_{\infty}=25 \mathrm{~km} \mathrm{~s}^{-1}$, so we obtain an interstellar Alfvén Mach number $M_{\mathrm{A} \infty} \approx 0.72$. Assuming an inner Alfvén Mach number of about 0.52, the inner magnetic field in the vicinity of the $x$-axis becomes about $4 \mu \mathrm{G}$. Taking the relation for the inner magnetic field, Eq. (100) and $d_{1}=10 \mathrm{AU}$, we are able to calculate the transformation, using Eqs. (86) and (91):

$$
\begin{aligned}
\alpha^{\prime}(A)= & \frac{1}{\sqrt{1-M_{A \infty}^{2}}}\left(\frac{B_{1}}{B_{\infty}} \tanh \frac{\frac{A}{\sqrt{1-M_{\mathrm{A} \infty}^{2}} B_{\infty}}-y_{1}}{d_{1}}\right. \\
& \left.-\frac{B_{1}}{B_{\infty}} \tanh \frac{\frac{A}{\sqrt{1-M_{\mathrm{A} \infty}^{2}} B_{\infty}}+y_{1}}{d_{1}}\right),
\end{aligned}
$$

where for $B_{1}$ we used the definition given by Eq. (99):

$$
B_{1}=\frac{1}{2}\left(B_{\infty}-\alpha^{\prime}(y=0) B_{S \infty}\right)=\frac{B_{\infty}}{2}\left(1-\frac{\sqrt{1-M_{\mathrm{A} \infty}^{2}}}{\sqrt{1-M_{\mathrm{A}, \mathrm{i}}^{2}}}\right) .
$$

The results for the different parameters are shown in Figs. 13-17, in Figs. 13 and 14 we plotted the $x$ - and $y$-components of the magnetic field in units of $5 \mu \mathrm{G}$. Towards the termination shock, the $x$-component grows especially around the $x$-axis because it is approaching the mapped magnetic monopole. The jumps due to the current sheet are of the order $1 \mu \mathrm{G}$. The $y$-component of the magnetic field is not symmetric with respect to the $x$-axis. Instead, a gradient arises due to the monopole. The contribution of the $y$-component to the total magnetic field strength is,

\footnotetext{
${ }^{6}$ The extreme value of the magnetic field is considered too high by P. Frisch, but preferred by Cox \& Helenius (2003).
} 


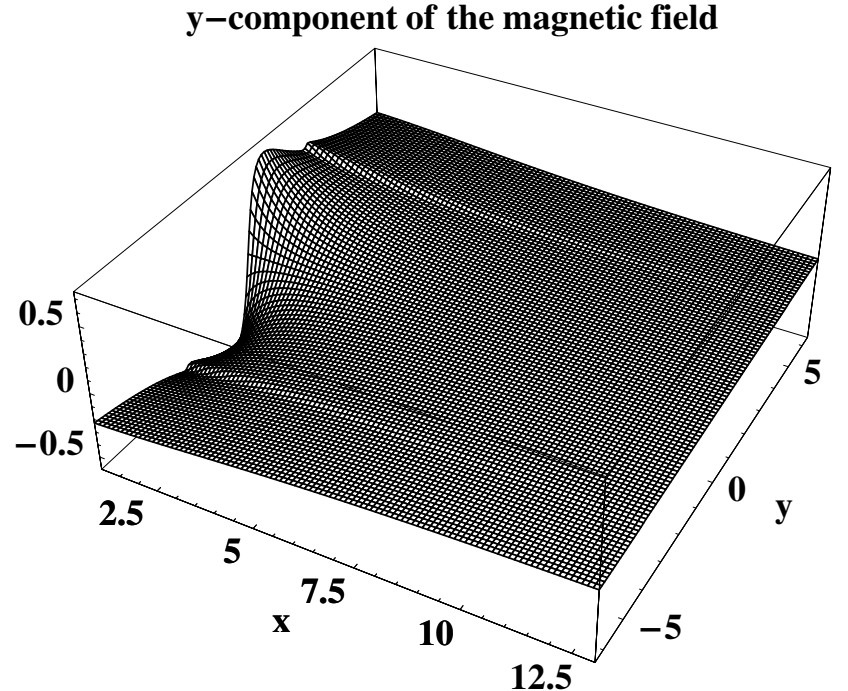

Fig. 14. The $y$-component of the magnetic field also shows a strong increase in its absolute value towards the termination shock region.

\section{Total strength of the magnetic field}

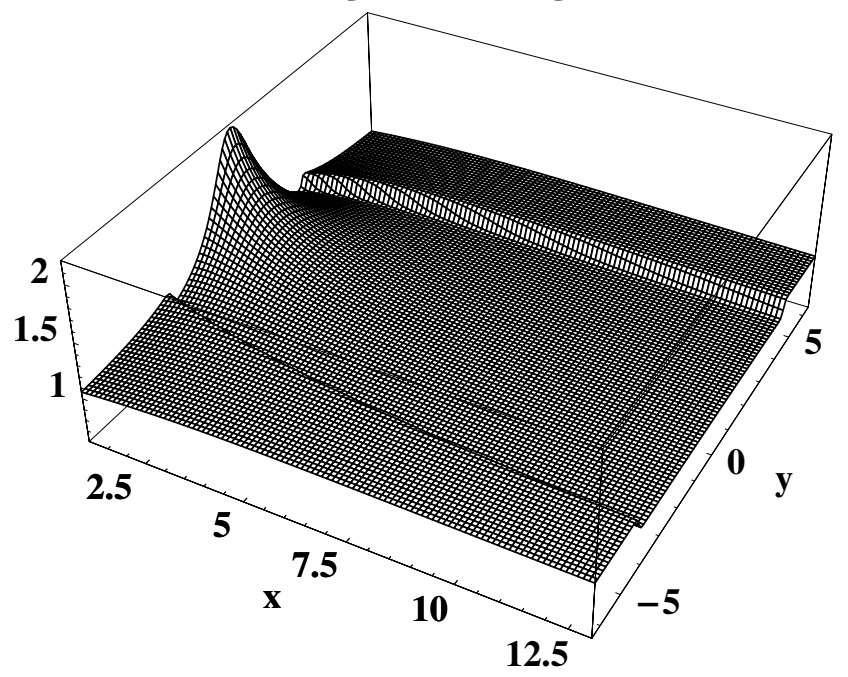

Fig. 15. Total strength of the magnetic field. The dominant contribution is from the $x$-component while, the contribution of the $y$-component is almost negligible.

however, very small, as can be seen in Fig. 15 (compared with Fig. 14). Figure 16 shows the strength of the current density. It is only different from zero at the locations of the current sheets where it shows steep gradients. In the last figure (Fig. 17) we display the behaviour of the Alfvén Mach number in the tail. It shows a strong gradient at the locations of the current sheets. This gradient even increases towards the termination shock.

\section{Discussion and conclusions}

We present a method for calculating nonlinear MHD equilibria with an incompressible field-aligned flow. This method is applied to the scenario of a flow of interstellar plasma around the plasma bubble of a strong magnetized stellar wind. We use the classical method of conformal mapping of flows around an obstacle as the starting point of our calculations.

We exclude violent structures (shocks) of flows in order to concentrate on the study of (i) the geometry of the contact current density

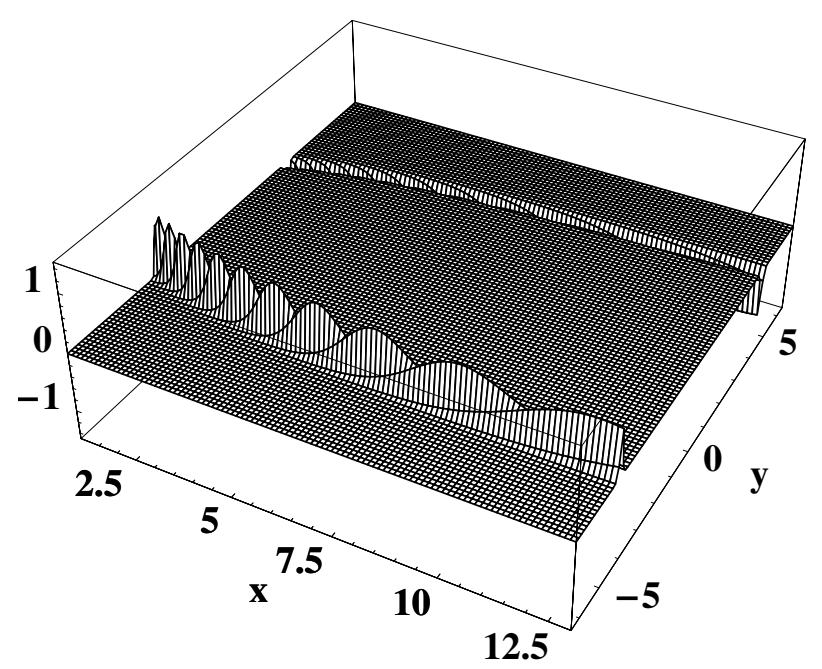

Fig. 16. The strength of the current density increases steeply towards the current sheets that have a width of $10 \mathrm{AU}$. The wavy shape of the peak current density is not physical but an artefact due to numerics. The current is normalized to units of $2.65 \times 10^{-17} \mathrm{Am}^{-2}$.

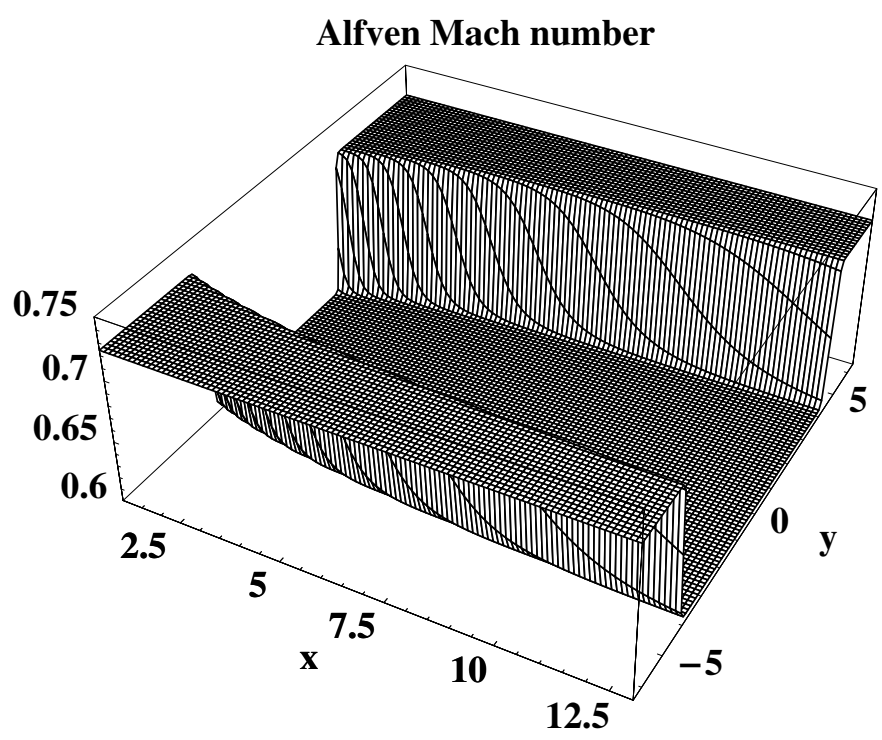

Fig. 17. This plot shows the Mach number and its strong gradient across the heliopause.

surface, (ii) the surface currents that are coupled to the inner and outer magnetic fields and the Alfvén Mach number as boundary conditions. The advantage of such a method is its high flexibility in modelling the tail of stellar winds and the surrounding interstellar medium wind. What we need at least is information on the singular points (stagnation- and magnetic neutral points), their numbers, and their orders. Hence, it would be better if in situ measurement of the magnetic field structure could be made. Within the next decade, this is only possible for our own astrosphere, the heliosphere.

In this paper, we restricted ourselves to thin nonsingular current sheets that have the special shape of a Harris-sheet or z-pinch configuration. The validity can only be justified within a multi-fluid theory or, better, within the framework of kinetic plasma theory and a detailed knowledge of the plasma environment in astrotails. Again, this aspect will be studied best observationally (in the next decade) for our heliosphere. 
As an improvement, additional current sheet structures should be taken into account, as e.g. the heliospheric current sheet is believed to extend beyond the heliospheric termination shock (see e.g. Pogorelov et al. 2004).

Our future aims are to use nonlinear static MHD equilibria as original equilibria, where the correlation between magnetic neutral points will be much more complex. In addition, we have to find mappings with corresponding boundary conditions. Another important point is the symmetry we have taken into account: symmetry of such configurations can be broken easily by an angle between the magnetic field and the probable flow direction in the vicinity of the heliosphere (see e.g. Frisch 1993). Such symmetry breaking will probably exclude an axially symmetric treatment of the problem. Nevertheless axially symmetric static equilibria can, in priciple, be used. In the case of pole-on counterflows of magnetized stellar winds, axial symmetry should be applied.

For further investigation, transformations should be used that allow for transitions from sub- to super-Alfvénic flows perpendicular to the magnetic field lines, as briefly described in Gebhardt \& Kiessling (1992). Their application is much more complicated, as it is necessary to introduce four Euler potentials for the representation of the velocity and the magnetic field.

Acknowledgements. This project was partly supported by the grant of the project No. 98030 of the PECS programme. D.H.N. thanks M. Karlicky for helpful discussions.

\section{References}

Arnold, V. I. 1992, Ordinary Differential Equations (Berlin: Springer) Baranov, V. B., \& Krasnobaev, K. V. 1971 Cosmic Res., 9, 620
D’haeseleer, W. D., Hitchon, W. N. G., Callen, J. D., \& Shohet, J. L. 1990, Flux Coordinates and Magnetic Field Structure (Berlin: Springer), 100

Chandrasekhar, S. 1956, ApJ, 124, 232

Cox, D. P., \& Helenius, L. 2003, ApJ, 583, 205

Fahr, H.-J., \& Neutsch, W. 1983, A\&A, 118, 57

Fahr, H.-J., Grezedzielski, S., \& Ratkiewicz, R. 1988, Ann. Geophys., 6, 337

Fahr, H.-J., Fichtner, H., \& Scherer, K. 1993, A\&A, 277, 249

Fahr, H.-J., Neutsch, W., \& Lay, G. 1982, Mitteil. Astron. Gesells., 57, 294

Frisch, P. C. 1993, ApJ, 407, 198

Frisch, P. C. 2004, Adv. Space Res., 34, 20

Gebhardt, U., \& Kiessling, M. 1992, Phys. Fluids B, 4, 1689

Goedbloed, J. P., \& Lifschitz, A. 1997, Phys. of Plasmas, 4, 3544

Grad, H., \& Rubin, H. 1958, Proc. 2nd Int. Conf. Peaceful Uses of Atomic Energy (Geneva: United Nations), 31, 190

Harris, E. G. 1962, Il Nuovo Cimento, 23, 115

Hameiri, E. 1998, Phys. Plasmas, 5, 3270

Imai, I. 1960, Rev. Mod. Phys., 32, 992

Kruskal, M. D., \& Kulsrud R. M. 1958, Phys. Fluids, 1, 265

Linde, T., et al. 1998, J. Geophys. Res., 103(A2), 1889

Lovelace, R. V. E., Mehanian, C., Mobarry, C. M., \& Sulkanen, M. E. 1986, ApJS, 62, 1

Nerney, S., Suess, S. T., \& Schmahl, E. J. 1991, A\&A, 250, 556

Neutsch, W., \& Fahr, H.-J. 1983, MNRAS, 202, 735

Parker, E. N. 1961, ApJ, 134, 20

Petrie, G. J. D., \& Neukirch, Th. 1999, Geophys. Astrophys. Fluid Dyn., 91, 269

Pogorelov, N. V., Zank, G. P., \& Ogino, T. 2004, ApJ, 614, 1007

Reitmann, V. 1996, Reguläre und Chaotische Dynamik (Leipzig: Teubner)

Scherer, K., Fahr, H.-J., \& Ratkiewicz, R. 1994, A\&A, 287, 219

Schindler, K. 1972, in Earth's Magnetospheric Processes, ed. B. M. McCormac (Dordrecht: Reidel), 200

Schindler, K. 1979, Space Sci. Rev., 23, 365

Smith, E. J. 2001, J. Geophys. Res., 106, 15819

Suess, S., \& Nerney, S. T. 1992, in Solar wind seven, ed. E. Marsch, \& R. Schwenn (Pergamon Press), 281

Suess, S. T., \& Nerney, S. T. 1990, J. Geophys. Res., 95, 6403

Tsinganos, K. C. 1981, ApJ, 245, 764

Tsinganos, K. C. 1982, ApJ, 259, 832

Webb, G. M., Brio, M., \& Zank, G. P. 1994, J. Plasma Phys., 52, 141 
D. H. Nickeler et al.: MHD flows in astrotails, Online Material $p 1$

\section{Online Material}




\section{Appendix A: Derivation of the transformation equations}

All solenoidal fields (i.e. vector fields with vanishing divergence) can be described locally by means of two scalar functions $f$ and $g$

$\boldsymbol{B}=\boldsymbol{\nabla} f \times \nabla g$.

Applying the scalar product $\boldsymbol{\nabla} f$ or $\boldsymbol{\nabla} g$, it follows that

$\boldsymbol{B} \cdot \boldsymbol{\nabla} f=0, \quad$ and $\quad \boldsymbol{B} \cdot \boldsymbol{\nabla} g=0$.

Since the potentials $f$ and $g$ are constant on field lines, besides the pressure in magnetohydrostatic equilibria $P_{S}$, we can understand $P$ as a function of $f$ and $g$ (i.e. intersections of $f=$ const and $g=$ const are field lines). We now want to consider the magnetohydrostatic equations with $\boldsymbol{B}_{S}=\boldsymbol{\nabla} f \times \boldsymbol{\nabla} g$, where $\boldsymbol{B}_{S}$ is the magnetic field of a known static MHD equilibrium. With the aforementioned equations, it follows that

$$
\boldsymbol{\nabla} P_{S}=\frac{1}{\mu_{0}}(\boldsymbol{\nabla} \times \boldsymbol{B}) \times \boldsymbol{B},
$$

and with $\boldsymbol{\nabla} P_{S}=\frac{\partial P_{S}}{\partial f} \boldsymbol{\nabla} f+\frac{\partial P_{S}}{\partial g} \boldsymbol{\nabla} g$,

we can extract the equations of motion

$$
\begin{aligned}
\frac{\partial P_{S}}{\partial f} & =\nabla g \cdot \nabla \times(\nabla f \times \nabla g), \\
-\frac{\partial P_{S}}{\partial g} & =\nabla f \cdot \nabla \times(\nabla f \times \nabla g) .
\end{aligned}
$$

The scalar potentials are often called Euler potentials. Using unmatched Euler potentials $\alpha$ and $\beta$, with $f=f(\alpha, \beta)$ and $g=g(\alpha, \beta)$, it follows from adopting the functional determinant or Poisson brackets defined as

$[f, g]_{\alpha, \beta}=\frac{\partial f}{\partial \alpha} \frac{\partial g}{\partial \beta}-\frac{\partial f}{\partial \beta} \frac{\partial g}{\partial \alpha}$,

that the following relation holds:

$\boldsymbol{B}_{S}=\boldsymbol{\nabla} f \times \boldsymbol{\nabla} g=[f, g]_{\alpha, \beta} \boldsymbol{\nabla} \alpha \times \boldsymbol{\nabla} \beta:=[f, g]_{\alpha, \beta} \boldsymbol{B}$.

Therefore,

$$
\begin{aligned}
& \mu_{0} \boldsymbol{\nabla} P_{S}=(\boldsymbol{\nabla} \times(\boldsymbol{\nabla} f \times \boldsymbol{\nabla} g)) \times(\boldsymbol{\nabla} f \times \boldsymbol{\nabla} g) \\
& =\left(\nabla \times[f, g]_{\alpha, \beta} \nabla \alpha \times \nabla \beta\right) \times[f, g]_{\alpha, \beta} \nabla \alpha \times \nabla \beta \\
& =\left(\boldsymbol{\nabla}[f, g]_{\alpha, \beta} \times \boldsymbol{\nabla} \alpha \times \boldsymbol{\nabla} \beta\right) \times[f, g]_{\alpha, \beta}(\boldsymbol{\nabla} \alpha \times \boldsymbol{\nabla} \beta) \\
& +\left([f, g]_{\alpha, \beta} \boldsymbol{\nabla} \times(\boldsymbol{\nabla} \alpha \times \boldsymbol{\nabla} \beta)\right) \times[f, g]_{\alpha, \beta}(\boldsymbol{\nabla} \alpha \times \boldsymbol{\nabla} \beta) \\
& =\left(\frac{1}{2} \nabla[f, g]_{\alpha, \beta}^{2} \times(\nabla \alpha \times \nabla \beta)\right) \times(\nabla \alpha \times \nabla \beta) \\
& +[f, g]_{\alpha, \beta}^{2} \boldsymbol{\nabla} \times(\boldsymbol{\nabla} \alpha \times \boldsymbol{\nabla} \beta) \times(\boldsymbol{\nabla} \alpha \times \boldsymbol{\nabla} \beta) \\
& =[f, g]_{\alpha, \beta}^{2} \boldsymbol{\nabla} \times(\boldsymbol{\nabla} \alpha \times \boldsymbol{\nabla} \beta) \times(\boldsymbol{\nabla} \alpha \times \boldsymbol{\nabla} \beta) \\
& +\underbrace{\left(\frac{1}{2} \nabla[f, g]_{\alpha, \beta}^{2} \cdot(\nabla \alpha \times \nabla \beta)\right)}_{\equiv 0}(\nabla \alpha \times \nabla \beta) \\
& -\frac{1}{2}(\nabla \alpha \times \nabla \beta)^{2} \nabla[f, g]_{\alpha, \beta}^{2} \\
& =[f, g]_{\alpha, \beta}^{2} \boldsymbol{\nabla} \times(\boldsymbol{\nabla} \alpha \times \boldsymbol{\nabla} \beta) \times(\boldsymbol{\nabla} \alpha \times \boldsymbol{\nabla} \beta) \\
& -\frac{1}{2}(\nabla \alpha \times \nabla \beta)^{2} \nabla[f, g]_{\alpha, \beta}^{2}
\end{aligned}
$$

If we identify the equilibrium magnetic field with

$\boldsymbol{\nabla} \alpha \times \nabla \beta \equiv \boldsymbol{B}$

the sum of thermal and ram pressure with

$P_{S} \equiv \Pi \equiv P+\rho|\boldsymbol{v}|^{2} / 2$,

and the corresponding equilibrium current density of the stationary equilibrium with

$\boldsymbol{\nabla} \times(\boldsymbol{\nabla} \alpha \times \nabla \beta) \equiv \mu_{0} \boldsymbol{j}$

we find

$\boldsymbol{\nabla} \Pi=\frac{1}{\mu_{0}}[f, g]_{\alpha, \beta}^{2}(\boldsymbol{j} \times \boldsymbol{B})-\frac{1}{2 \mu_{0}} \boldsymbol{B}^{2} \boldsymbol{\nabla}[f, g]_{\alpha, \beta}^{2}$.

We recognize the identical form of the last term on the right side of Eq. (A.13) with the right-hand side of the equation of motion (Eq. (A.14)), which is

$\nabla \Pi=\frac{1}{\mu_{0}}\left(1-M_{\mathrm{A}}^{2}\right)(\boldsymbol{j} \times \boldsymbol{B})-\frac{1}{2 \mu_{0}} \boldsymbol{B}^{2} \boldsymbol{\nabla}\left(1-M_{\mathrm{A}}^{2}\right)$,

where we identify the Poisson brackets of Eq. (A.13) with the Alfvén Mach numer dependent expression in Eq. (A.14). Then it follows that

$1-M_{\mathrm{A}}^{2} \equiv[f, g]_{\alpha, \beta}^{2}>0$.

For non-canonical transformations, the Poisson brackets has a non-constant value or a value different from unity. For canonical transformations, the Mach number is zero, or for a constant and non-unity value of the Poisson brackets, the Mach number turns out to be constant. It is therefore possible to map known solutions of the magnetohydrostatic equations with the help of non-canonical transformations into stationary solutions with a sub-Alfvénic flow. For $M_{\mathrm{A}}>1$, we find an analogy with the equations of incompressible stationary hydrodynamics (see Gebhardt \& Kiessling 1992). Application of the same transformation as in the case of the sub-Alfvénic equilibrium results in the same magnetic field and therefore in the same electric current. On the other hand, for Mach numbers with $M_{\mathrm{A}}^{2} \geq 2$, we cannot find any sub-Alfvénic Mach number or solution.

The pressure is going to be inverted with respect to the subAlfvénic pressure to become:

$\Pi_{\text {super-Alfvenic }}=\Pi_{H}-\Pi_{\text {sub-Alfvenic }}$.

Here, $\Pi_{H}$ is a background pressure, which guarantees that the thermal pressure stays positive everywhere and fullfills the physical conditions (as e.g. $v_{S}>v_{\mathrm{A}}$ ), as well as the boundary conditions.

As shown above, it is possible to map known solutions of the magnetohydrostatic equations via the non-canonical transformations into stationary solutions with sub-Alfvénic flow. If there exists a non-canonical transformation $f=f(\alpha, \beta)$ and $g=g(\alpha, \beta)$ or $\alpha=\alpha(f, g)$ and $\beta=\beta(f, g)$, then those stationary 
fields are given by

$$
\begin{aligned}
\boldsymbol{B} & =\boldsymbol{\nabla} \alpha \times \boldsymbol{\nabla} \beta=\frac{1}{[f, g]_{\alpha, \beta}} \nabla f \times \nabla g=\frac{1}{\sqrt{1-M_{\mathrm{A}}^{2}}} \boldsymbol{B}_{S}, \\
\boldsymbol{v} & =\frac{M_{\mathrm{A}}(\alpha(f, g), \beta(f, g))}{\sqrt{\mu_{0} \rho(\alpha(f, g), \beta(f, g))}} \boldsymbol{B}=\frac{M_{\mathrm{A}}(f, g)}{\sqrt{\mu_{0} \rho(f, g)}} \boldsymbol{B} \\
& =\frac{M_{\mathrm{A}}}{\sqrt{\left(1-M_{\mathrm{A}}^{2}\right) \mu_{0} \rho}} \boldsymbol{B}_{S}, \\
P & =\Pi(f, g)-\frac{M_{\mathrm{A}}^{2}}{2}|\boldsymbol{B}|^{2}=P_{S}(f, g)-\frac{M_{\mathrm{A}}^{2}}{2 \sqrt{1-M_{\mathrm{A}}^{2}}}\left|\boldsymbol{B}_{S}\right|^{2}, \\
\rho & =\rho(f, g) .
\end{aligned}
$$

\section{Appendix B: Trajectories}

If $f$ is a flux function, then $f$ is constant on field lines. For $\Delta A=$ $-\mu_{0} \mathrm{~d} P / \mathrm{d} A=J(A)$ and with the help of the implicit function given by $\chi=\chi(x, y)$, where $\chi_{0}=\chi(x, y)=$ const, it follows that $A(\chi)=$ const. The equation $\chi=\chi(x, y)=$ const therefore describes the bundle of the field lines of $A(\chi)$.

The equation

$\Delta A=\frac{\mathrm{d}^{2} A}{\mathrm{~d} \chi^{2}}(\nabla \chi)^{2}+\frac{\mathrm{d} A}{\mathrm{~d} \chi} \Delta \chi=-\mu_{0} \mathrm{~d} P / \mathrm{d} A=J(A(\chi))$

can therefore be regarded as the differential equation for $A$ as a function of $\chi$, if we have

$\frac{\partial\left(|\nabla \chi|^{-2} \Delta \chi, \chi\right)}{\partial(x, y)}=0$

in the case of a vanishing current function (i.e. a potential field), i.e. for $J(A)=0$. This results in a non-linear partial differential equation.

\section{B.1. Radial magnetic fields}

For $\Delta A=-\mu_{0} \mathrm{~d} P / \mathrm{d} A=J(A)=0$ and for radial trajectories, it follows that, for $y / x=$ const on straight lines, the function $A(\chi)=$ const. $\chi$ is thereby regarded as a function of $x$ and $y$, with $\chi(x, y):=y / x$. Application of the Laplace operator on $A(\chi(x, y))$ results in

$$
\begin{aligned}
\Delta A= & A^{\prime \prime}(\chi)\left(\frac{y^{2}}{x^{4}}+\frac{1}{x^{2}}\right)+A^{\prime}(\chi) \frac{2 y}{x^{3}}=0 \\
& \Rightarrow A^{\prime \prime}(\chi)\left(\chi^{2}+1\right)+2 A^{\prime}(\chi) \chi=0 .
\end{aligned}
$$

The second row of Eq. (B.3) can be expressed as

$$
\frac{\mathrm{d}}{\mathrm{d} \chi}\left(A^{\prime}(\chi)\left(1+\chi^{2}\right)\right)=0 \text {. }
$$

Integration leads to

$A(\chi)=$ const $\cdot \arctan (\chi)+A_{0}$,

with $A_{0}$ as a constant of integration. The function $A(\chi)$ can be expressed in the framework of a $2 \mathrm{D}$ multipole expansion with the help of the imaginary part of the line current, i.e. with the radial magnetic field (represented by the imaginary part of the complex logarithm). 